\title{
Diffusion Generated Motion for Grain Growth in Two and Three Dimensions
}

\author{
Matt Elsey, Selim Esedoḡlu, and Peter Smereka \\ Department of Mathematics, University of Michigan, Ann Arbor, MI 48109 \\ E-mail: melsey@umich.edu, esedoglu@umich.edu, psmereka@umich.edu
}

April 25, 2009

\begin{abstract}
An efficient algorithm for accurately simulating curvature flow for large networks of curves in two dimensions and surfaces in three dimensions on uniform grids is proposed. This motion arises in the technologically important problem of simulating grain boundary motion in polycrystalline materials. In this formulation grains are zero level sets of signed distance functions. Curvature motion is achieved by first diffusing the signed distance functions followed by a reinitialization step. A technique is devised to allow a single signed distance function to represent many grains allowing the simulation of a very large number (hundreds of thousands) of grains using modest computational hardware.
\end{abstract}

Keywords: diffusion-generated motion; mean curvature flow; grain growth.

\section{Introduction}

A polycrystalline material contains many crystallites (often called grains), differentiated by varying orientation. These materials are very commonplace, including most metals and ceramics. The properties of the microscale polycrystalline structure affect macroscale properties of these materials, such as fracture strength and conductivity. As such, understanding the statistics of the microscale structure is of great interest to materials scientists. One important model consists of grains moving with a normal velocity equal to curvature with grain boundaries meeting at $120^{\circ}$ at triple points (for example, see Mullins [16] or Hillert [8]). Much work has focused on obtaining various statistics of grains evolving in this manner since such quantities may hold the key to important macroscopic properties. In order to obtain robust results, large scale simulations with hundreds of thousands to millions of grains would be ideal. This is a challenging computational problem, especially in three dimensions. We propose and demonstrate a new algorithm for large-scale simulations of this evolution, expanding on the work in [3].

According to the well-known model of Mullins [16], grain boundaries evolve with normal velocity given by

$$
v_{n}=\mu \gamma \kappa
$$

where $\mu$ denotes the boundary mobility, $\gamma$ the surface tension, and $\kappa$ the mean curvature of the interface between grains. In many cases (e.g. the isothermal annealing of pure metals), the mobility and surface tension may be taken to be constant, so that the normal velocity of the interface (a curve in two dimensions and a surface in three dimensions) is proportional to mean curvature. We consider the simplest, yet still important, case: that of equal surface tensions for each grain. We set $\mu \gamma=1$ for convenience. As shown in [17] and [28], this normal speed arises as gradient descent for the energy

$$
E=\sum_{k<\ell}\left(\text { length of } \Gamma_{k \ell}\right)
$$

where $\Gamma_{k \ell}$ is the boundary between grain $k$ and grain $\ell$ (and length is replaced by area in three dimensions). The standard boundary condition for this problem is the Herring angle condition, which for equal surface tensions states that triple junctions must meet at angles of $120^{\circ}$. This angle condition arises naturally from the algorithms used in this paper, as shown in [3]. 
The algorithm we use in this paper is based on distance function based diffusion generated motion developed by Esedoglu, Ruuth, and Tsai [3] in which signed distance functions to interfaces are convolved with Gaussian kernels to generate a variety of geometric motions, including multi-phase motion by mean curvature, in an unconditionally stable manner. These algorithms are variants of the original threshold dynamics scheme of Merriman, Bence, and Osher [15] in which characteristic functions are diffused to generate mean curvature motion. Replacing characteristic functions in [15] with signed distance functions allows algorithms in [3] to attain good accuracy on modest sized uniform grids with no need for adaptive refinement. In [15] the authors also consider multi-phase mean curvature motion and propose an algorithm based on representing each phase by a level set function and evolving them via the standard level set PDE for mean curvature motion; see also [26]. A detailed discussion of the differences between the algorithm in [3] (an extended version of which will be developed and used here) and those in [15] can be found in [3].

The problem of simulating networks of grains moving by curvature flow has attracted much attention over the decades and many different computational approaches have been proposed. We feel that the algorithm presented here has advantages over previous formulations. Indeed, we are able to perform well-resolved simulations of grain networks in both two and three dimensions on a scale significantly larger than previously reported in the literature. We can easily simulate more than 250000 grains in two dimensions, and 100000 grains in three dimensions.

In previous work, grain boundary networks moving by curvature flow have been simulated by front tracking techniques in both two (e.g. [10]) and three dimensions (e.g. [25]). Computational efficiency is the big advantage of this approach since all the computational resources are concentrated on the interface. A fundamental difficulty of this approach is managing the plethora of topological changes that can occur as grains disappear. These methods must explicitly detect and handle each topological change by some selection of rules and require that the triple point condition be maintained separately. Furthermore, it is difficult to check if edges (in two dimensions) or surfaces (in three dimensions) cross using explicit methods. In three dimensions, it is a particularly difficult task to enumerate the ways in which two explicitly represented surfaces might meet. Even more difficult, if not practically impossible, would be checking to see if any such collisions occur. Using such representations in practice requires making assumptions about the types of topological changes that can typically occur. These assumptions may leave out important transitions or allow for nonphysical artifacts such as the interpenetrating of phase boundaries. In two dimensions, it is expected (though not proven) that boundary networks under pure curvature motion change topology only through junction collisions, greatly simplifying the class of interactions possible. However, no such expectation is held in three dimensions. Even in the two-phase case, one phase can pinch off and split into two pieces. In two dimensions, the addition of bulk energy terms to the energy will also generally result in more complicated topological changes.

The phase-field formulation (e.g. $[5,7,9,12,23]$ ) will ameliorate these difficulties, but introduce a problem of its own. In this approach, a phase function is evolved for each grain and the grain boundary is a level set of the phase function. These methods naturally handle the aforementioned difficulties associated with topological changes but require the phase function to have a sharp transition layer at the grain boundary. It is crucial that this layer be fully resolved in order to accurately approximate curvature flow. For example, Kim et al. [9] report that they need at least 6 grid points in the transition layer to achieve acceptable accuracy. This indicates that a typical grain size must have something like 25 grid points per dimension to be even marginally resolved - a serious limitation to the accurate simulation of a large number of grains. We show that, using the approach described in this paper, our grains are well-resolved with 10 grid points per dimension, and we can follow them as they shrink down to about 4 or 5 grid points per dimension with a few percent relative error. Similar difficulties are present using threshold dynamics $[14,15,19,21]$. Threshold dynamics methods can become "stuck" on uniform grids in regions where the interface moves slowly (although this situation can be remedied via adaptive refinement; see e.g. [19,20]).

Another approach is to use a Potts model via kinetic Monte Carlo techniques (e.g. [1]). This is essentially a different model and its connection with curvature flow is a difficult question. Finally we mention that there are level set techniques, different than those present here, that could also be employed to tackle this problem (e.g. [4,28]).

The algorithm used in this paper is able to capture many of the advantages of the above methods with few of the disadvantages. Our method represents the grain boundaries implicitly using a signed distance function thereby achieving subgrid accuracy on a uniform mesh. Our method naturally handles topological changes and naturally imposes the Herring angle conditions (i.e. $120^{\circ}$ ) at junctions. Therefore, we capture the advantages of the phase field method without the disadvantage of needing to resolve a transition layer. In addition, the algorithms proposed in this paper are unconditionally stable. We 
have developed a technique in which sufficiently separated grains are represented by the same distance function (something similar was done for a phase field method in [12]). In addition, some of the computational work can be confined to narrow band near the grain boundaries. In this way we keep some of the advantage of the front-tracking formulations.

In this work, we implement the algorithm introduced in [3] (more fully described in Section 2). We then present results from a large two-dimensional simulation (initially containing over 150000 grains), and preliminary results from a three-dimensional simulation (beginning with over 15000 grains) in Section 3 (detailed three-dimensional results with over 100000 grains will be published separately). We conclude by presenting an extension to the algorithm in Section 4 which allows for the inclusion of bulk energy terms into the energy functional for which our evolution constitutes gradient descent dynamics.

\section{Algorithm}

The signed distance function-based diffusion generated algorithm for motion by mean curvature of multiple phases as proposed in [3] is reproduced below. The evolution generated by this algorithm is proven to generate motion by mean curvature and to satisfy the symmetric Herring angle condition at triple junctions [3] in 2D (and hence also along triple lines in 3D).

We shall first describe the the algorithm present in [3] using the following notation: The $k$ th grain at time $t=n \Delta t$ will be denoted as $\Sigma_{k}^{n}$ where $\Delta t$ is the time step. The signed distance function from the boundary of $\Sigma_{n}^{k}$ is denoted as $d_{n}^{k}$. Our sign convention is such that $d_{n}^{k}>0$ for points in $\Sigma_{n}^{k}$. Further we suppose that there are $N$ grains. The algorithm devised in [3] is then given by:

1. Given the initial sets $\Sigma_{1}^{0}, \ldots, \Sigma_{N}^{0} \subset \mathbb{R}^{m}$ construct the corresponding signed distance functions $d_{k}^{0}$ (i.e. $\Sigma_{k}^{0}=\left\{x: d_{k}^{0}(x)>0\right\}$ ).

For $n=0, \ldots, n_{\max }$, perform steps $2-4$.

2. Form the convolutions: $A_{k}(x):=K_{\Delta t} * d_{k}^{n}$ for $k=1, \ldots, N$, where $K_{\Delta t}$ is

$$
K_{\Delta t}=G_{\Delta t} \quad \text { or } \quad K_{\Delta t}=\frac{1}{4}\left(4 G_{\frac{3}{2} \Delta t}-G_{3 \Delta t}\right)
$$

and $G_{\Delta t}$ is the fundamental solution of the heat equation:

$$
G_{\Delta t}(x)=\frac{1}{(4 \pi \Delta t)^{\frac{m}{2}}} e^{-\frac{|x|^{2}}{4 \Delta t}} .
$$

3. Construct $B_{k}(x)$ for $k=1, \ldots, N$ to remove overlaps and vacuums from the convolution step:

$$
B_{k}(x)=\frac{1}{2}\left(A_{k}(x)-\max _{\ell}\left\{A_{\ell}(x): \ell \neq k\right\}\right)
$$

4. Construct the updated signed distance function $d_{k}^{n+1}(x)$ for $k=1, \ldots, N$ according to

$$
d_{k}^{n+1}(x)=\operatorname{Redist}\left(B_{k}(x)\right) .
$$

Remarks. The operation Redist $(B(x))$ means construct a signed distance function from the zero level set of $B(x)$. Formally $G_{\Delta t}$ and $K_{\Delta t}=\frac{1}{4}\left(4 G_{\frac{3}{2} \Delta t}-G_{3 \Delta t}\right)$ have the same order of accuracy but the spatial truncation error of the second one is devoid of terms involving derivatives of curvature; see [3] for details. The redistribution or projection step 3 of the algorithm above is the same as in the threshold dynamics case [15] and is motivated by the well-known phase-field formulation (e.g. [2,7]) of the problem.

As stated the algorithm uses one distance function per grain. Clearly if the grains are not immediate neighbors, then a single distance function can serve to represent many grains. Further, if we demand only that grains which are sufficiently far apart share the same distance function, then potential interactions that could occur during the convolution step will be negligible.

\subsection{Extension}

Here we present an extension of the algorithm from the previous section that allows one to use the same distance function for multiple grains. The setting for this algorithm is slightly different than the previous 
one. We begin as before, namely with $N$ grains $\Sigma_{k}^{0}$ with $k=1, \ldots, N$, but initialize only $M$ signed distance functions, $d_{\ell}^{0}$ with $\ell=1, \ldots, M$, where $M<N$. These $d_{\ell}^{0}$ have the property that they are the signed distance function for a collection of disjoint grains, and the union of these collections consists of all the grains. As the algorithm proceeds, it must check to be sure that this disjointness property is maintained. If it appears that it is about to fail (i.e. two distinct grains in one of the collections become too close), various grains will need to be reassigned to different distance functions and if need be a new distance function will be introduced. We call this operation swapping. A crucial point is that $M \ll N$ unless one considers some pathological initial conditions. Even then, since the evolutions considered here are regularizing with a preference towards grains with small isoperimetric ratios, $M$ is expected and observed to be fairly small at subsequent times during the evolution.

1. Given the initial sets $\Sigma_{1}^{0}, \ldots, \Sigma_{N}^{0}$ construct $M$ functions, $d_{k}^{0}$ so that each is the signed distance function for a collection $\Xi_{k}$ of disjoint grains so that $\cup_{k=1}^{M} \Xi_{k}=\cup_{k=1}^{N} \Sigma_{k}$. For $n=0, \ldots, n_{\max }$, perform steps $2-5$.

2. Form the convolutions: $A_{k}(x):=K_{\Delta t} * d_{k}^{n}$ for $k=1, \ldots, M$

3. Construct $B_{k}(x)$ for $k=1, \ldots, M$ to remove overlaps and vacuums from the convolution:

$$
B_{k}(x)=\frac{1}{2}\left(A_{k}(x)-\max _{\ell}\left\{A_{\ell}(x): \ell \neq k\right\}\right)
$$

4. Construct the signed distance function $d_{k}^{n+\frac{1}{2}}(x)$ for $k=1, \ldots, M$ according to

$$
d_{k}^{n+\frac{1}{2}}=\operatorname{Redist}\left(B_{k}(x)\right) \text {. }
$$

5. If necessary swap appropriate grains between signed distance functions to ensure that all the grains associated to given signed distance function remain well separated. Denote the resulting signed distance functions as $d_{k}^{n+1}$.

\subsubsection{Details}

We now describe the steps of the above algorithm in more detail in the fully discrete setting. For convenience, the formulas are written down in the 2D setting, but extend trivially to all dimensions.

Convolution. We define the convolution kernels $G_{\Delta t}$ and $K_{\Delta t}$ in terms of the space-discretized solution to the heat equation $u_{t}=u_{x x}+u_{y y}$. Suppose the grid discretizes $[0,1]^{2}$, with equal grid spacing $(\Delta x=\Delta y)$. Let $u_{i, j}(t)$ be the space-discretized approximation to $u(x, y, t)$ at $(i \Delta x, j \Delta y, t)$. Using centered differencing in space, we obtain:

$$
\frac{d}{d t} u_{i, j}(t)=\frac{1}{\Delta x^{2}}\left(\left(u_{i+1, j}-2 u_{i, j}+u_{i-1, j}\right)+\left(u_{i, j+1}-2 u_{i, j}+u_{i, j-1}\right)\right) .
$$

Apply the discrete Fourier transform in space to obtain,

$$
\frac{d}{d t} \widehat{u}_{r, s}=\frac{2}{\Delta x^{2}}(\cos (2 \pi s \Delta x)+\cos (2 \pi r \Delta x)-2) \widehat{u}_{r, s}
$$

Given initial data $\widehat{u}_{r, s}(t)$, this ODE has solution $\widehat{u}_{r, s}(t+\Delta t)$

$$
\widehat{u}_{r, s}(t+\Delta t)=\widehat{u}_{s, r}(t) \exp \left(\frac{-2 \Delta t}{\Delta x^{2}}(2-\cos (2 \pi r \Delta x)-\cos (2 \pi s \Delta x))\right) .
$$

Therefore the discrete heat equation (1) has solution $u_{i, j}(t+\Delta t)=u_{i, j} *\left(G_{\Delta t}\right)_{i, j}$ where $*$ denotes the discrete convolution and $\left(G_{\Delta t}\right)_{i, j}$ is defined via its discrete Fourier transform:

$$
\left(\widehat{G}_{\Delta t}\right)_{r, s}=\exp \left(\frac{-2 \Delta t}{\Delta x^{2}}(2-\cos (2 \pi r \Delta x)-\cos (2 \pi s \Delta x))\right) .
$$

Finally, we implement a Richardson extrapolation-like procedure to improve the accuracy of the kernel, (as described in [3]), and define:

$$
K_{\Delta t}=\frac{1}{3}\left(4 G_{\frac{3}{2} \Delta t}-G_{3 \Delta t}\right)
$$


Comparison. The convolution step gives diffusion generated motion along simple interfaces, but may create overlaps or vacuums at junctions where multiple interfaces meet. To enforce the desired no overlap / no vacuum condition, we apply a comparison step to obtain the updated level set functions

$$
B_{k}(x)=\frac{1}{2}\left(A_{k}(x)-\max \left\{A_{\ell}: \ell \neq k\right\}\right),
$$

satisfying the condition

$$
\sum_{k=1}^{m} H\left(B_{k}(x)\right)=1
$$

for all $x$, where $H$ denotes the Heaviside function. This procedure ensures that the symmetric Herring angle condition is maintained at all triple points [3].

Redistancing. At each time step, we need to compute the signed distance function $d_{k}$ to a union $\Xi_{k}$ of disjoint grains; we need the distance function only in a tubular neighborhood of the boundary $\partial \Xi_{k}$ of $\Xi_{k}$. The width of the tubular neighborhood is dependent on the time step $\Delta t$. We make use of a two-phase redistancing algorithm that depends only on the input values $B_{k}(x)=\frac{1}{2}\left(A_{k}(x)-\max \left\{A_{\ell}: \ell \neq k\right\}\right)$ at grid points within two grid points of the interface. For the remainder of the discussion of redistancing, we drop the subscript $k$ for convenience, as each set is updated independently of the others.

Define the set of boundary points $\beta$ to be

$$
\beta=\left\{(i, j):\left(\left|\operatorname{sgn}\left(B_{i+1, j}\right)-\operatorname{sgn}\left(B_{i-1, j}\right)\right|+\mid \operatorname{sgn}\left(B_{i, j+1}\right)-\operatorname{sgn}\left(B_{i, j-1}\right)\right) \mid>0\right\},
$$

where $B_{i, j}=B(i \Delta x, j \Delta y)$ and

$$
\operatorname{sgn}(x)= \begin{cases}1 & \text { if } x>0 \\ 0 & \text { if } x=0 \\ -1 & \text { if } x<0\end{cases}
$$

These boundary values are set initially to respect the condition that $|\nabla d| \equiv\left|\nabla d_{j+\frac{1}{2}}^{k}\right|=1$, while moving the interface as little as possible. Specifically, we set

$$
d_{i j}=\frac{B_{i j}}{\left|\nabla B_{i j}\right|}, \quad \forall(i, j) \in \beta
$$

Typically, the centered difference approximation is appropriate for $\left|\nabla B_{i j}\right|$, but does not work well on small grains. See Figure 1 for an illustration in one dimension. The solid line is the exact signed distance function to the thick bar shown at the bottom of the plot. The dashed line shows the centered difference approximation to the gradient of the signed distance function at the indicated point. Upwind differencing is only first-order accurate in general, but gives a more accurate value for the gradient at this point. We define

$$
\begin{gathered}
\left|\nabla B_{i j}\right|_{1}=\sqrt{\left(\frac{B_{i+1, j}-B_{i-1, j}}{2 \Delta x}\right)^{2}+\left(\frac{B_{i, j+1}-B_{i, j-1}}{2 \Delta y}\right)^{2}}, \\
\left|\nabla B_{i j}\right|_{2}=\sqrt{\left(\max \left(\frac{\left|B_{i+1, j}-B_{i, j}\right|}{\Delta x}, \frac{\left|B_{i, j}-B_{i-1, j}\right|}{\Delta x}\right)\right)^{2}+\left(\max \left(\frac{\left|B_{i, j+1}-B_{i, j}\right|}{\Delta y}, \frac{\left|B_{i, j}-B_{i, j-1}\right|}{\Delta y}\right)\right)^{2}},
\end{gathered}
$$

and define

$$
\left|\nabla B_{i j}\right|= \begin{cases}\left|\nabla B_{i j}\right|_{1} & \text { if } \frac{1}{2}\left|\nabla B_{i j}\right|_{2} \leq\left|\nabla B_{i j}\right|_{1} \leq 2\left|\nabla B_{i j}\right|_{2}, \\ \left|\nabla B_{i j}\right|_{2} & \text { otherwise. }\end{cases}
$$

We fix the values $d_{i j}$ for all $(i, j) \in \beta$, and first generate a first-order in space accurate approximation of the signed distance function using fast sweeping as described in [24,27]. Then we perform an iterative second-order accurate method (described in [18]) for a limited number of iterations on this output. The input $B(x)$ may be far from a distance function near junctions. Performing the fast sweeping initially allows us to perform only a limited number of iterations with the second-order method, which is the most time-intensive part of the algorithm.

Swapping. The swapping step allows each signed distance function to store many grains safely. Without this step, it would be necessary to maintain each individual grain in a separate set to guarantee that coalescence could not occur. For example, in a calculation performed on a $4096^{2}$ grid, we begin with over 160000 grains and use only $M=32$ sets to track them all. The algorithm introduces new signed distance 


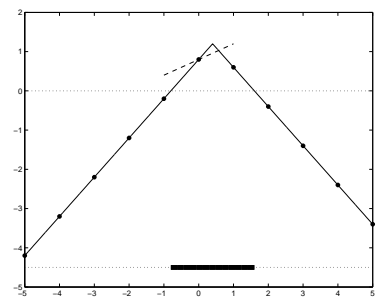

Figure 1: Failure of centered differencing on small grains. The thick black line indicates the set $\Sigma=\{x: d(x)>0\}$. The slope of the dashed line indicates the centered difference approximation to $|\nabla d|_{1}$ at the specified point. The upwind differencing finds $|\nabla d|_{2}=1$, the correct value for $|\nabla d|$.

(a)

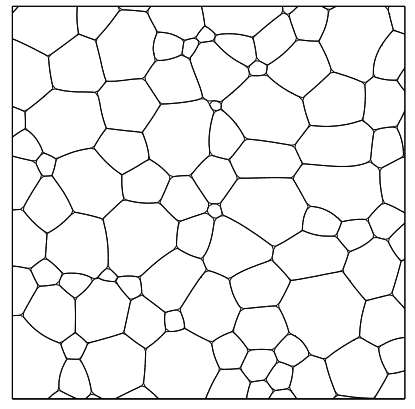

(b)

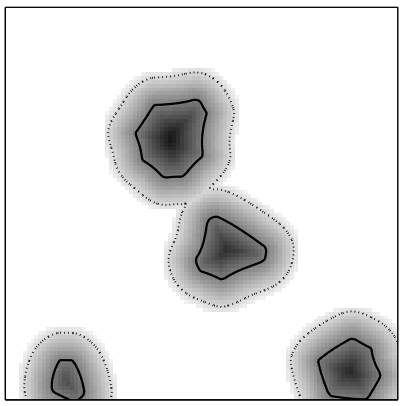

(c)

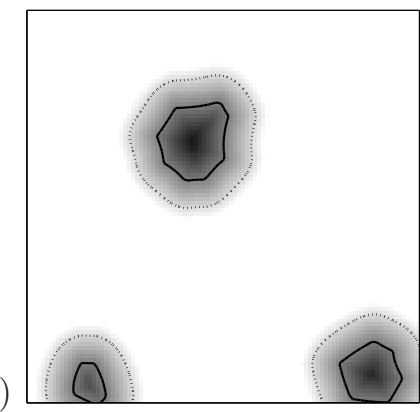

Figure 2: (a) Part of a grain pattern. (b) Overlay of the boundary of $d^{n+\frac{1}{2}}>0$ (solid line) and $d^{n+\frac{1}{2}}>-\tau$ (dotted line) on the signed distance function $d^{n+\frac{1}{2}}$. (c) Same for $d^{n+1}$ after a grain is removed and $d^{n+1}$ is recalculated.

functions if needed to ensure that inter-grain spacing is maintained. In our experience, the algorithm typically does not require more than $M=32$ sets in two dimensions, and not more than $M=64$ sets in three dimensions. As the grain network evolves we find it will rarely, if ever, introduce new signed distance functions. Without the savings of both memory and computational time permitted by this additional step (allowing the number of sets, $M$, to satisfy $M \ll N$, the total number of grains), such a large scale computation would be impossible.

Our approach, described below, is similar to that of Krill and Chen [12]. They reassign grains to prevent any particular grain from being maintained in the same set as any of it's nearest or second-nearest neighbors. We, instead, make sure that any two grains described by the same signed distance function are not too close (we will be more precise shortly). This distinction is significant for our algorithm, as spatial separation is key to prevent distinct grains, described by the same signed distance function, from interacting during the convolution step (the width of the kernel is of course related to the time step size, which can be large thanks to the unconditional stability of the proposed algorithms).

To describe this algorithm we must first outline some notation. First recall that the set $\Xi_{k}=\{x$ : $\left.d_{k}(x)>0\right\}$ corresponds to a collection of disjoint grains. These grains are the connected components of $\Xi_{k}$. We say that two grains, say, $\Sigma_{a}$ and $\Sigma_{b}$ in $\Xi_{k}$ are $\tau$ - close if their union is completely contained in the same connected component of $\left\{x: d_{k}(x)>-\tau\right\}$ (which is trivially checked by comparing the $(-\tau)$-super level set membership of any two grid points belonging to $\Sigma_{a}$ and $\left.\Sigma_{b}\right)$. We choose $\tau>0$ to be proportional to $\sqrt{\Delta t}$ to prevent distinct grains in $\Xi_{k}$ from interacting during the convolution step. In the simulations presented in Section 3 , we take $\tau \approx 6 \sqrt{\Delta t}$. See Figure 2 for illustration of the selection process.

Here we describe the new step in the algorithm in greater detail.

1. For $k=1, \ldots, M$, initialize $d_{k}^{n+1}=d_{k}^{n+\frac{1}{2}}$.

2. For all signed distance functions make list of all pairs of grains that are $\tau$ - close.

For each pair of grains, perform steps $3-7$.

3. Select the smaller grain from the pair and denote the signed distance function they are associated with as $k$. Let $d_{G}(x)$ be the signed distance function to the boundary of the selected grain and define the set $X=\left\{x: d_{G}(x)>-\tau\right\}$.

4. Find a set, $\Xi_{\ell}^{n+1}$, such that $\ell \neq k$ and $d_{\ell}^{n+1}(x) \leq-\tau \forall x \in X$. If such a set cannot be found, increment $M \leftarrow M+1$, initialize $d_{M}^{n+1}=-\tau$, and select $\ell=M$. 


\begin{tabular}{cccccc}
\hline Resolution & Iterations & $r\left(t^{\star}\right)$ & \% Error & Exact Redist Result & \% Error \\
\hline $8 \times 8$ & 7 & 0.143812 & $15.0493 \%$ & 0.068539 & $45.1687 \%$ \\
$16 \times 16$ & 15 & 0.124301 & $0.5595 \%$ & 0.112370 & $10.1040 \%$ \\
$32 \times 32$ & 30 & 0.123497 & $1.2022 \%$ & 0.123544 & $1.1649 \%$ \\
$64 \times 64$ & 60 & 0.123918 & $0.8653 \%$ & 0.124435 & $0.4520 \%$ \\
$128 \times 128$ & 120 & 0.124246 & $0.6110 \%$ & 0.124562 & $0.3501 \%$ \\
$256 \times 256$ & 240 & 0.124585 & $0.3323 \%$ & 0.124751 & $0.1996 \%$ \\
$512 \times 512$ & 480 & 0.124797 & $0.1627 \%$ & 0.124870 & $0.1043 \%$ \\
$1024 \times 1024$ & 960 & 0.124900 & $0.0800 \%$ & 0.124934 & $0.0532 \%$ \\
$2048 \times 2048$ & 1920 & 0.124952 & $0.0383 \%$ & 0.124966 & $0.0268 \%$ \\
\hline
\end{tabular}

Table 1: Convergence Check: Motion by curvature of a circle.

5. Add the grain to $\Xi_{\ell}^{n+1}$ by setting $d_{\ell}^{n+1}(x)=d_{G}(x) \forall x \in X$.

6. Remove this same grain from $\Xi_{k}^{n+1}$ by setting $d_{k}^{n+1}(x)=-\tau \quad \forall x \in X$.

7. Redistance $d_{k}^{n+1}$ and $d_{\ell}^{n+1}$ on the set $X$.

\section{$3 \quad$ Numerical Results}

In this section, two types of numerical results are presented. First, the convergence of our algorithm to exact solutions in two- and three-phase cases is displayed. Next, we examine the convergence in a multiphase case for which no exact solution is known. Finally, we demonstrate the quality of our algorithm on large data sets simulating normal grain growth using statistical measures such as average grain size and grain area distribution.

\subsection{Convergence to Exact Solutions in Two Phase Motion}

We begin by verifying that our algorithm accurately simulates two phase motion by mean curvature on the simplest examples in two and three dimensions: the circle and the sphere. In each case, the motion reduces to the simple ordinary differential equation,

$$
\dot{r}(t)=\kappa=\frac{-C}{r},
$$

where $C=1$ for the circle and $C=2$ for the sphere. Eq. (2) has the solution

$$
r(t)=\sqrt{r(0)^{2}-2 C t} .
$$

In our tests, we chose $r(0)=.25$. For the circle, we took as our stopping time $t^{\star}=3 / 128$, and for the sphere $t^{\star}=3 / 256$, so that the exact solution has $r\left(t^{\star}\right)=.125$. We iterate through time using a simple forward Euler step, and therefore expect to see linear convergence, despite using redistancing and convolution kernels that are second-order accurate in space. See Tables 1 and 2 for numerical results. The results labeled "Exact Redist Result" were obtained by replacing the distance function at the redistancing step by the exact distance function for a circle or sphere with the same 0 - level set at each step. We note that the linear convergence rate is strongly indicated by the exact redistancing results for resolutions $\geq 256 \times 256$ for the circle and $\geq 64 \times 64 \times 64$ for the sphere. Our redistancing technique causes some cancellations of error at low resolutions, but follows the linear convergence trend shown by the exact redistancing results well at higher resolutions.

\subsection{Convergence to Known Profile in Three Phase Motion}

In this test of three phase motion, we choose homogenous Neumann boundary conditions and consider a $T$-junction initial condition as shown in Figure 3. It was shown in [7] that there is an exact solution for this $T$ junction geometry consisting of a steady profile moving at constant speed. The profile is given for $0 \leq x \leq 0.5$ by:

$$
y(x, t)=\frac{3}{\pi} \log \left(\cos \left(\frac{\pi x}{3}\right)\right)-v t
$$




\begin{tabular}{cccccc}
\hline Resolution & Iterations & $r\left(t^{\star}\right)$ & $\%$ Error & Exact Redist Result & \% Error \\
\hline $8 \times 8 \times 8$ & 7 & 0.153862 & $23.0896 \%$ & 0.113474 & $9.2212 \%$ \\
$16 \times 16 \times 16$ & 15 & 0.128935 & $3.1484 \%$ & 0.120004 & $3.9964 \%$ \\
$32 \times 32 \times 32$ & 30 & 0.124040 & $0.7682 \%$ & 0.123044 & $1.5648 \%$ \\
$64 \times 64 \times 64$ & 60 & 0.123881 & $0.8951 \%$ & 0.124010 & $0.7923 \%$ \\
$128 \times 128 \times 128$ & 120 & 0.124289 & $0.5688 \%$ & 0.124481 & $0.4150 \%$ \\
$256 \times 256 \times 256$ & 240 & 0.124627 & $0.2988 \%$ & 0.124735 & $0.2121 \%$ \\
\hline
\end{tabular}

Table 2: Convergence Check: Motion by curvature of a sphere.

where $v$ is the velocity of the profile. Figure 3 shows the close agreement between the predicted profile where $v t$ was chosen to match the computed profile at $x=0$. These results were computed on a $128 \times 128$ grid. In (a), the predicted profile and computed profiles are indistinguishable. Parts (b), (c), and (d) zoom in successively on the final computed profile and the associated prediction. We see that the results differ by less than $10^{-3}$ for all $x$. The triangular "split" in the profile seen in the zoomed views is purely a visualization artifact.

\subsection{Spatial Convergence of Multiphase Motion}

There are no explicit solutions available for the evolution of a general grain pattern. To test convergence, we instead evolve the same initial condition for 100 iterations with fixed time step at various resolutions: $1024 \times 1024,512 \times 512,256 \times 256$, and $128 \times 128$.

We begin with the initial condition seen in Figure 4(a), which contains 154 grains. After 100 iterations, only 82 (83, at $128 \times 128$ resolution) grains remain. See Figure 4 (b). The $1024 \times 1024$ result is indistinguishable from the $512 \times 512$ result to the eye. We note that the visible differences at various resolutions are attributable to recent topological changes in the grain pattern. When the grain size is approximately equal to the grid size, we cannot hope to simulate the motion accurately. At the microscopic level, grains on this size scale tend to persist somewhat longer than they should (Figure 4(c)), though results agree very well in regions without recent topological changes (Figure 4(d)). The macroscopic parameters of the evolution (e.g. number of grains, average grain size, etc.) are unaffected by small discrepancies in the evolution at the scale of the grid size.

\subsection{Large-Scale Simulation of Normal Grain Growth in Two Dimen- sions}

We concern ourselves with the standard case, in which all interfaces move with normal velocity equal to the curvature of the interface. In future work we will investigate varying surface tensions and bulk energies. As previously shown in [3], this algorithm naturally imposes the symmetric angle condition (all triple junctions meet at $120^{\circ}$ angles).

We discretize the $[0,1] \times[0,1]$ domain with $\Delta x=\Delta y=1 / 4096$. We use periodic boundary conditions, natural as the interactions between grains are short-ranged. Our initial condition contains 166927 grains, and was obtained by constructing the Voronoi diagram for random points with a uniform distribution in the computational domain. We take 1500 time steps, at the end of which 11217 grains remain and the evolution of the system has slowed considerably. (An adaptive time stepping strategy would therefore be prudent, and is entirely feasible given the unconditional stability of our algorithms, but this was not carried out: All experiments in this paper were generated using uniform time steps). For the entire evolution, we maintain just 32 sets to track all the grains. For snapshots of the evolution, see Figure 5. These all show $1 / 64$ of the full grain pattern. Figure 6 shows the final full grain pattern. Earlier time steps contain too many grains for the full pattern to be viewable on a single page.

Recall that the energy of the system is given by

$$
E=\sum_{k<\ell}\left(\text { length of } \Gamma_{k \ell}\right)
$$

in two spatial dimensions (and by summing the interfacial area in three dimensions). In terms of the signed distance functions $d_{k}(x)$, this energy can be written in terms of the Dirac delta function, $\delta$, as

$$
E=\frac{1}{2} \sum_{k=1}^{M} \int_{\Omega} \delta\left(d_{k}(x)\right) d x .
$$



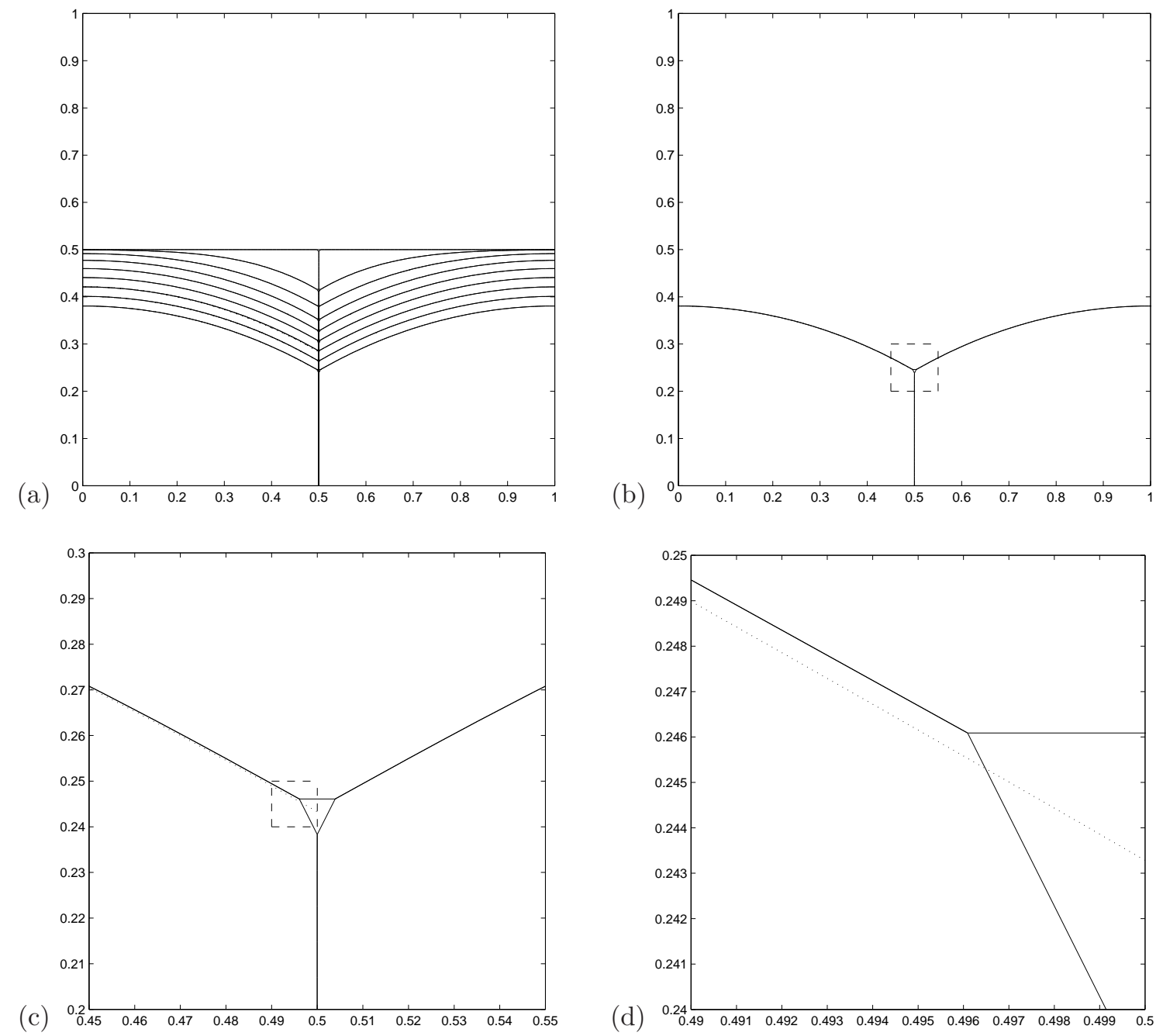

Figure 3: (a) Interface shown at various times in evolution (solid). The exact profile is overlaid (dotted) once a constant profile is attained. (b), (c), (d) Successive zoom in to the computed profile (solid) and exact profile (dotted). 
(a)
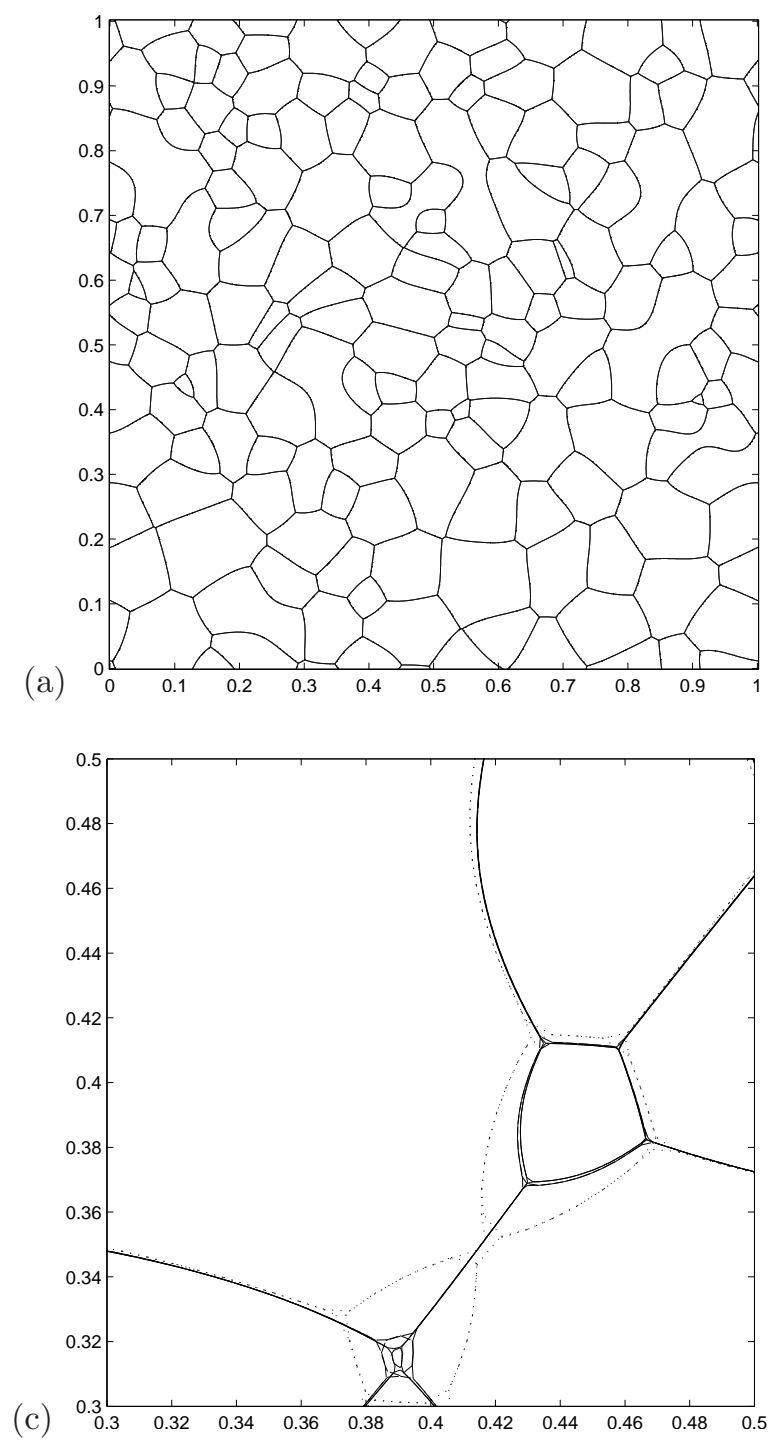

(b)

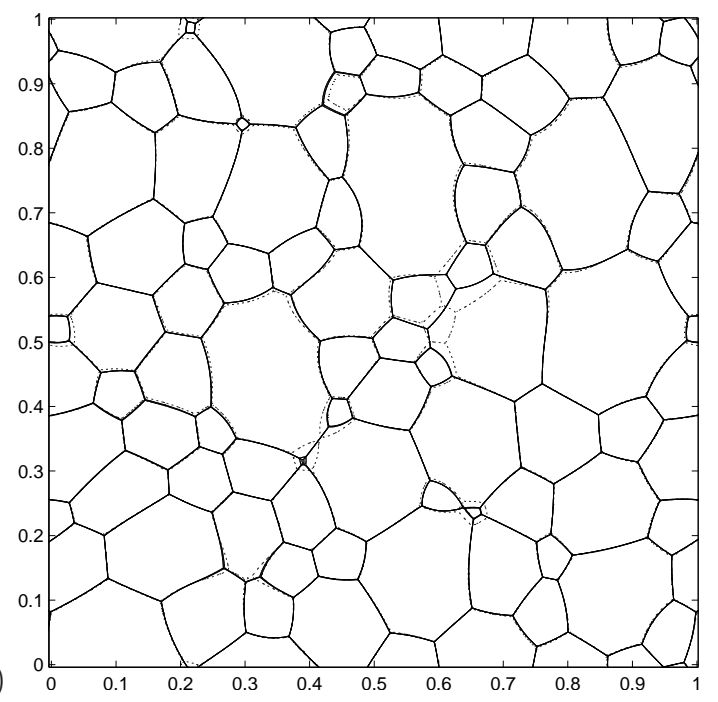

(d)

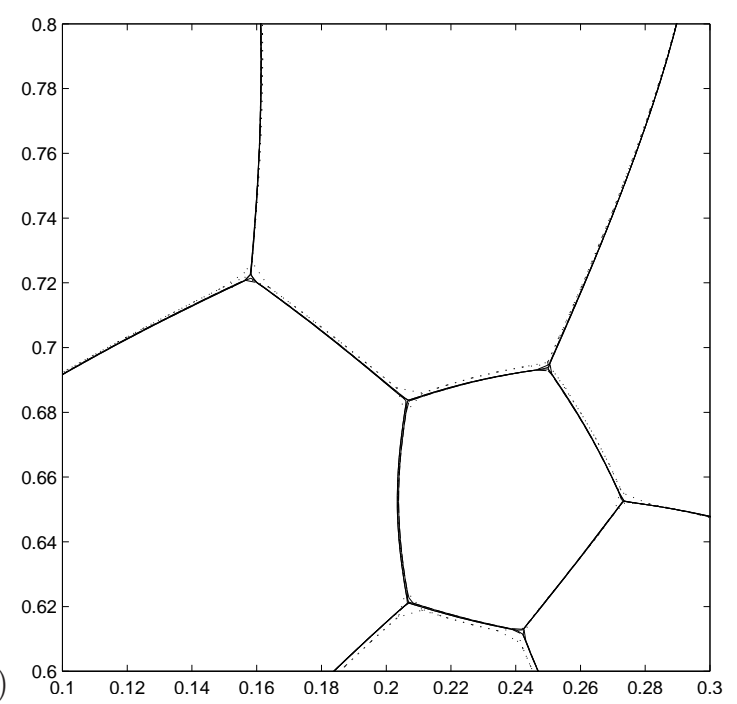

Figure 4: (a) Initial condition. (b) Overlay of $512 \times 512$ result (solid), $256 \times 256$ result (dashed), and $128 \times 128$ result (dotted). The results at $1024 \times 1024$ and $512 \times 512$ resolution are indistinguishable. Only at $128 \times 128$ are significant differences visible on parts of the grid, near recent topological changes. (c) and (d) zoom in on two parts of (b). 


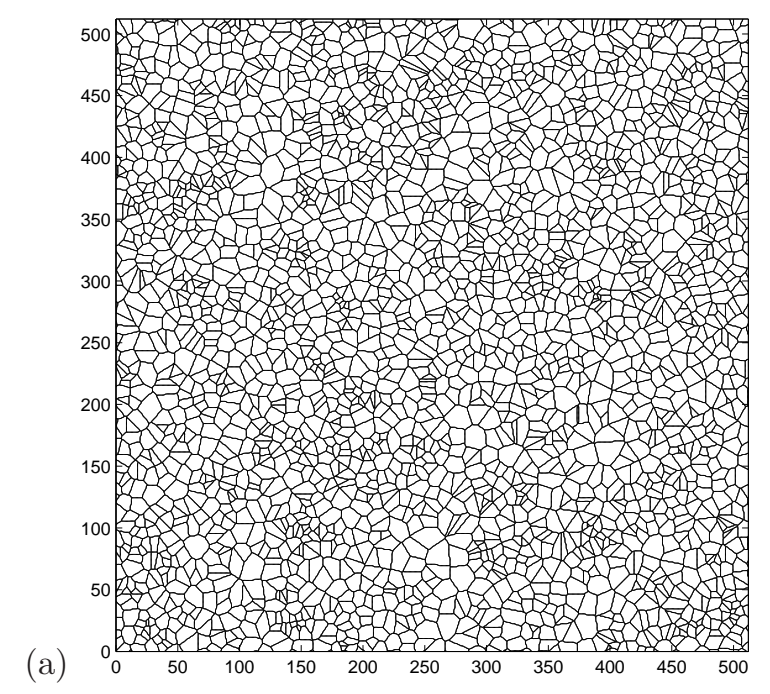

(b)
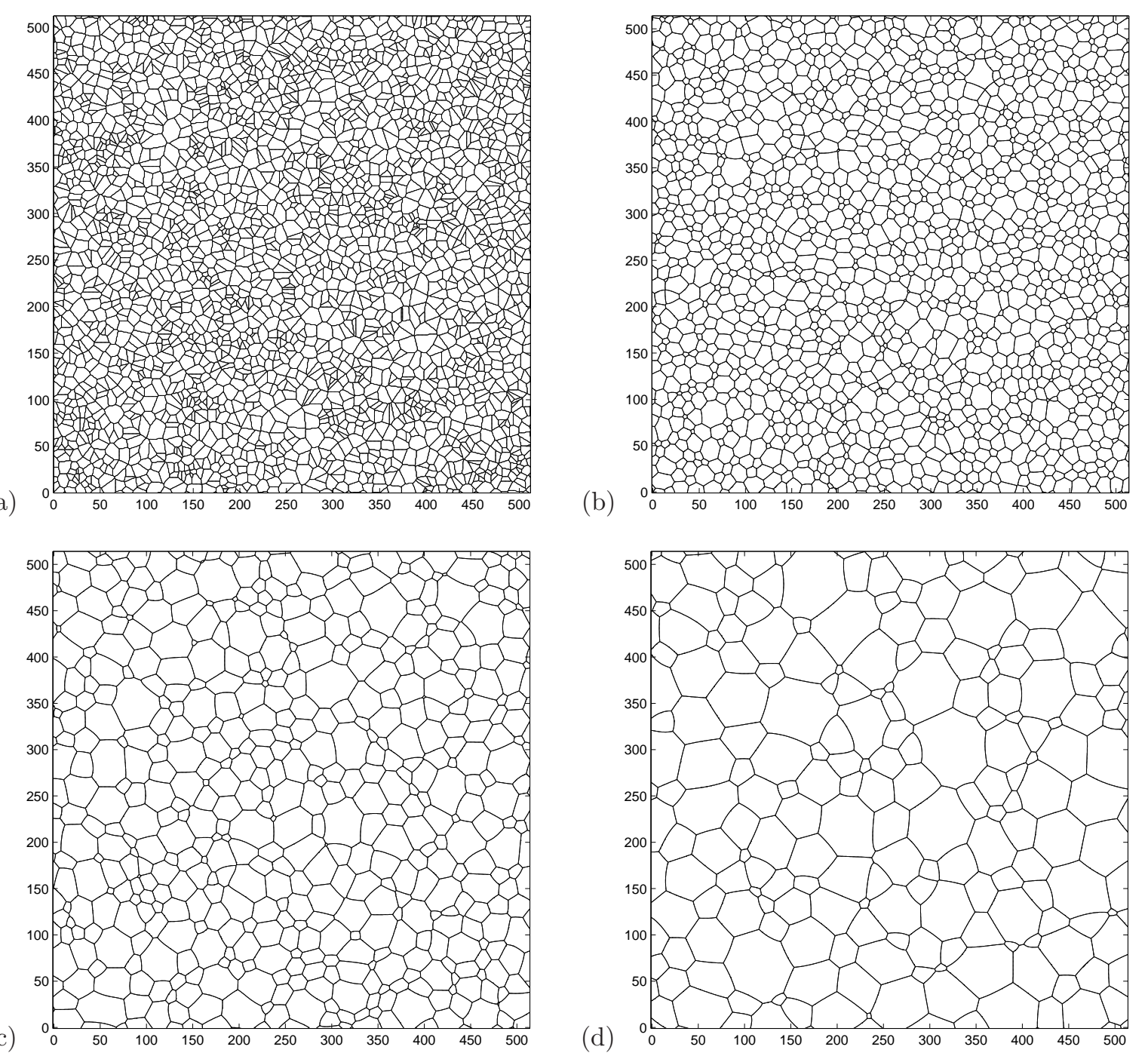

(d)

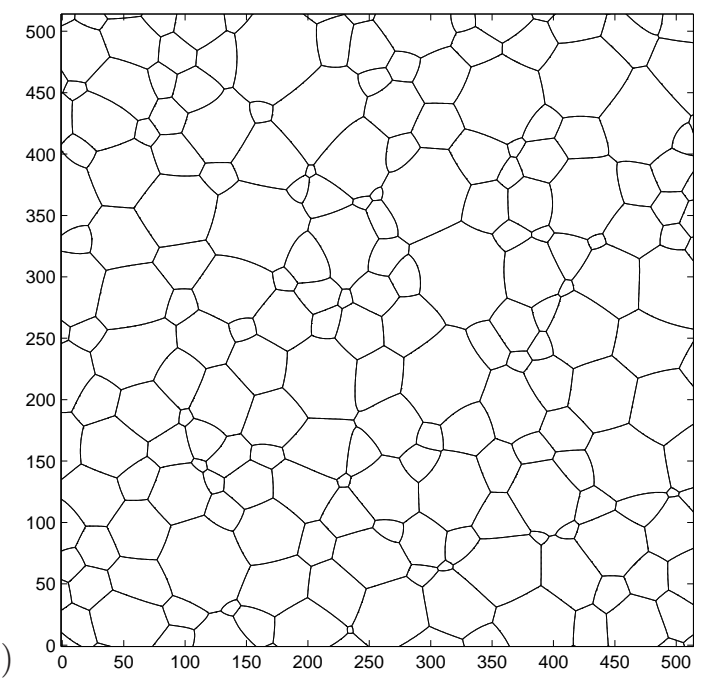

Figure 5: One section of the full $4096 \times 4096$ grid. (a) Initial condition, $N(0)=166927$, (b) after 100 iterations, $N(100)=97000$, (c) after 500 iterations, $N(500)=30842$, (d) after 1500 iterations, $N(1500)=11217$. 


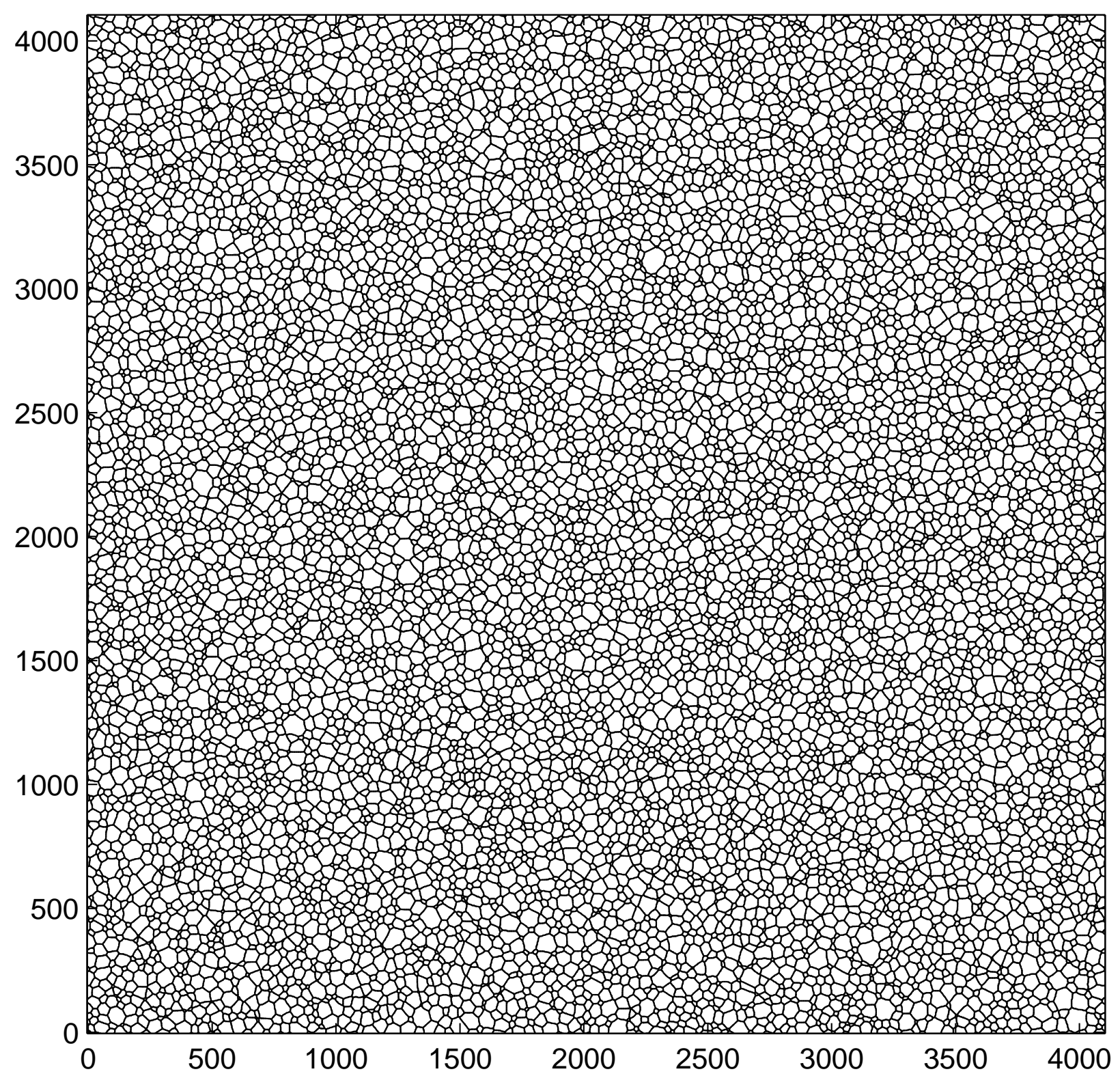

Figure 6: The full grain pattern after 1500 iterations for the simulation with $N(0)=166927$ grains initially. At the time shown, $N(1500)=11217$ grains remain. 


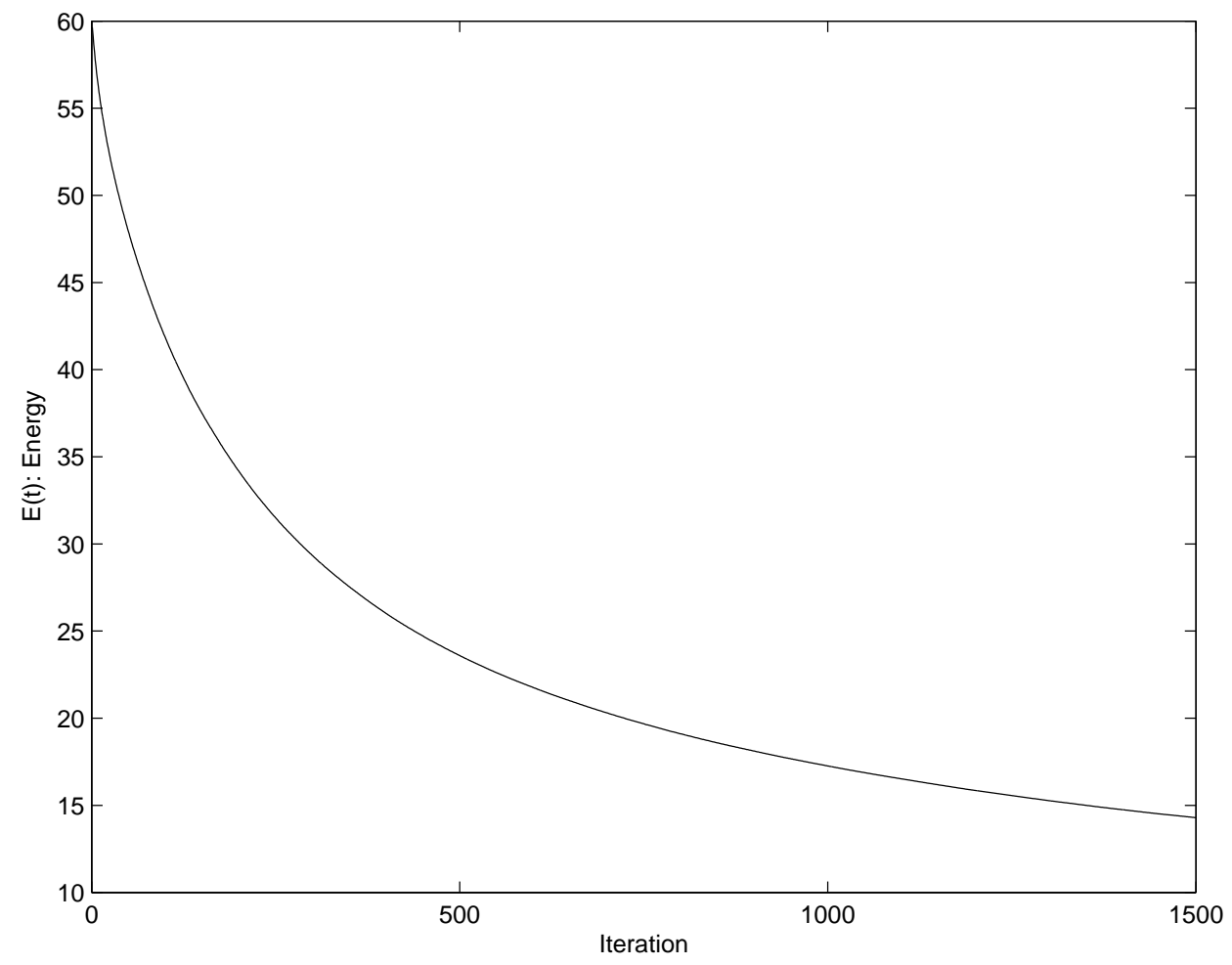

Figure 7: The energy $E$ decreases at every time step.

The factor $1 / 2$ arises since this formula counts interfaces two times. We can discretize $E$, in two space dimensions, as

$$
E=\frac{\Delta x^{2}}{2} \sum_{k} \sum_{i, j} \tilde{\delta}\left(d_{k}\left(x_{i}, y_{j}\right)\right) .
$$

We use a first-order discretization of the delta function, $\tilde{\delta}$, as proposed in [22]. The discrete version of Eq. (3) in three dimensions is similar. The energy, $E$, is evaluated at each iteration and found to be strictly decreasing at every time step. See Figure 7. Note that our method handles topological changes naturally, and that the energy of the system decreases even as over 150000 grains disappear throughout 1500 iterations.

Several analytical approaches predict the mean grain radius $\langle r\rangle$ to grow as $\langle r\rangle \approx C t^{1 / 2}$ (for example, see $[6,8,13])$. In normal grain growth, characterized by self-similarity of the distribution of $r /\langle r\rangle$, it immediately follows that the average grain area $\langle a\rangle$ is predicted to grow linearly as a function of time. We compute the average grain area as

$$
\langle a\rangle=\frac{1}{N(t)},
$$

where $N(t)$ is the number of grains surviving at time $t$, and see linear growth following a short relaxation time due to initial conditions. See Figure 8.

Two other measures of interest are the relative grain area distribution and the number of edges distribution, as defined in [11]. Let $G(n, t)$ be the proportion of grains with $n$ grain boundaries at time $t$, and $F(\xi, t) d \xi$ be the number of grains with relative area in $[\xi, \xi+d \xi]$ at time $t$, with $\xi=a /\langle a\rangle$. Normal grain growth is characterized by the self-similarity of $F$ as $t$ varies. See Figure 9 , which suggests that $F$ is approximately self-similar for $t \geq 300 \Delta t$. Figure 10 shows the evolution of the number of edges distribution.

\subsection{Three Dimensional Multiphase Motion by Curvature}

Our algorithm and its implementation extends with only minor modifications to three dimensions very naturally. We again consider the case in which all interfaces (surfaces) move with normal velocity equal 


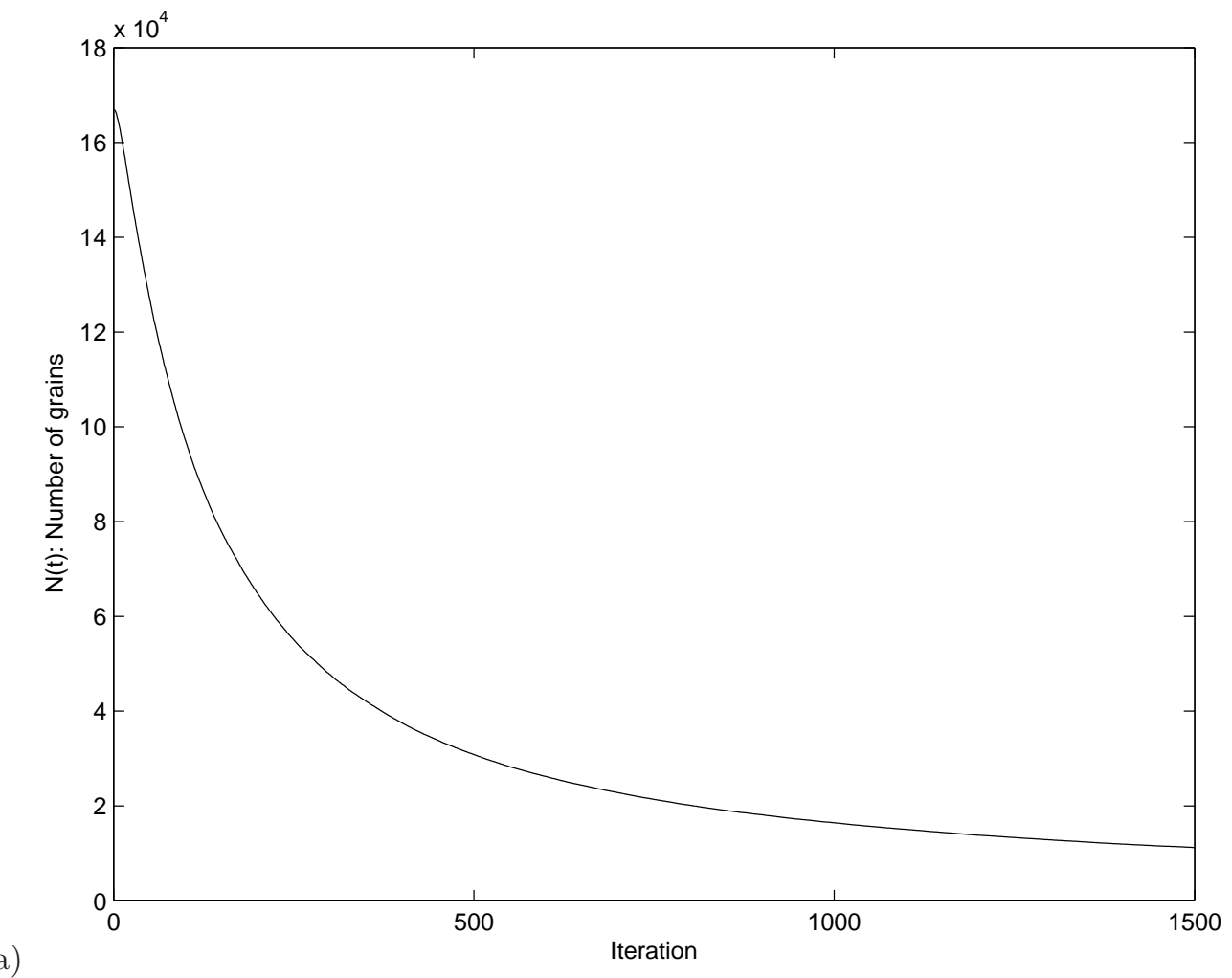

(a)

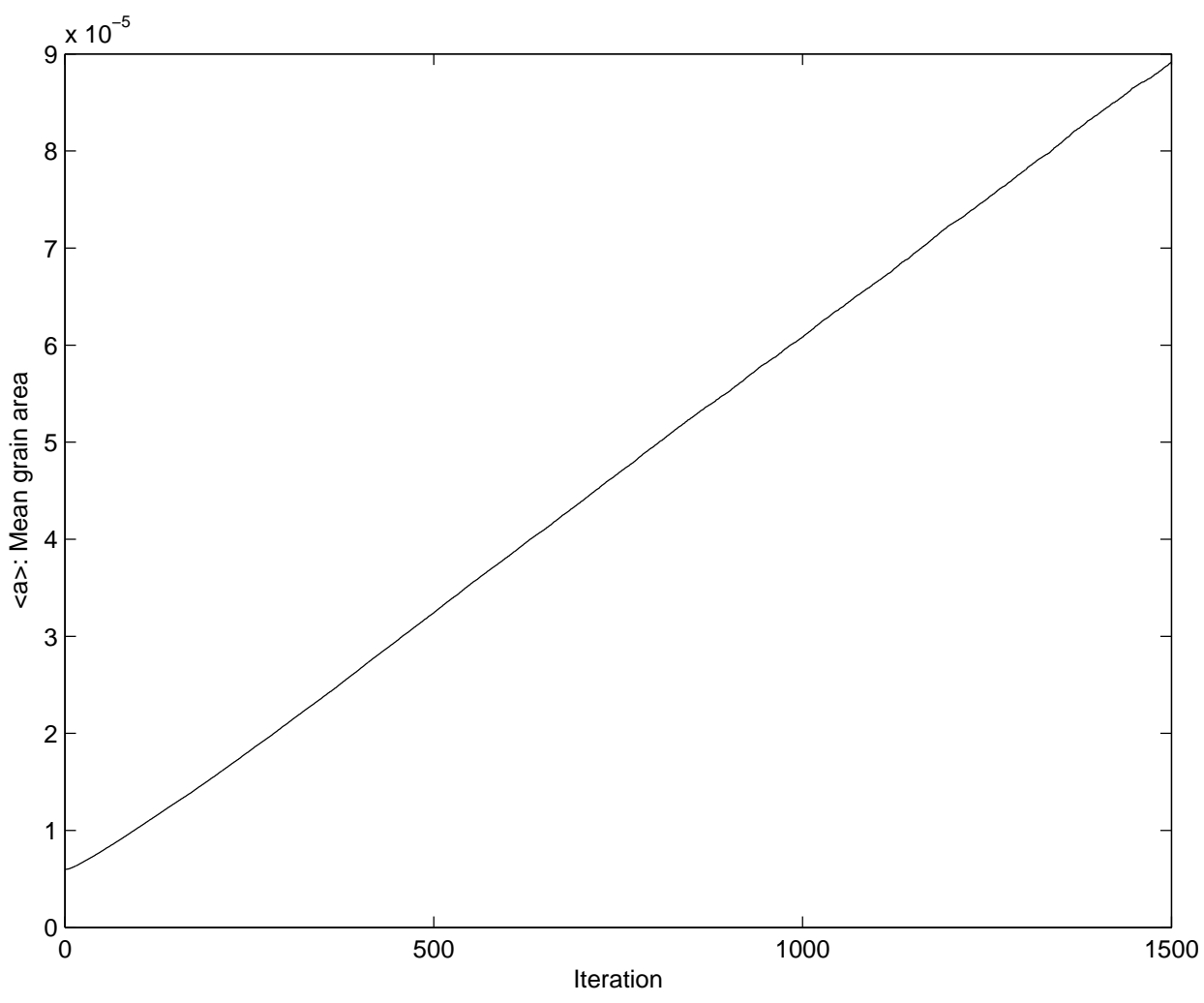

(b)

Figure 8: (a) The number of grains $N(t)$ decreases such that (b) average grain area $\langle a\rangle$ grows linearly. 


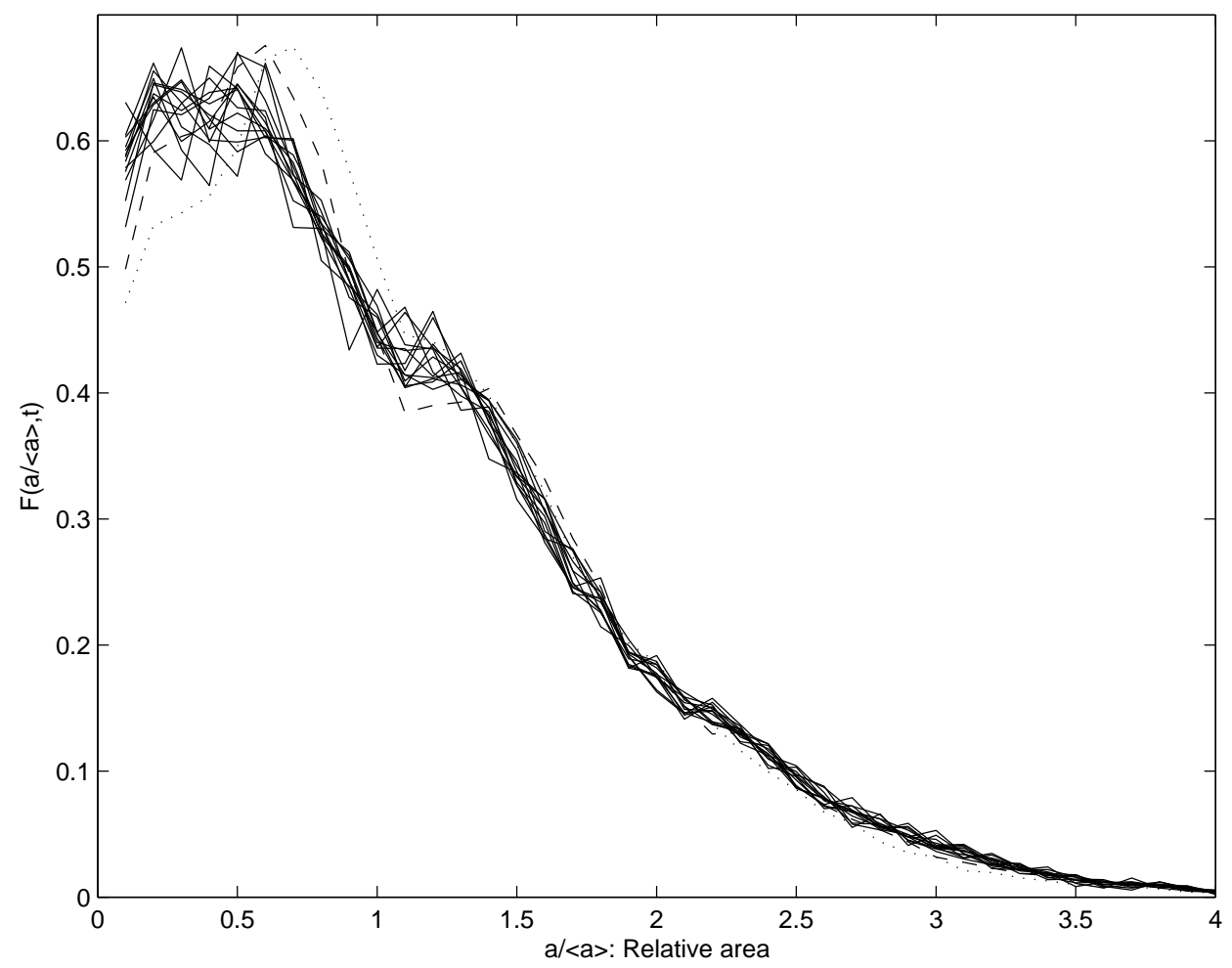

Figure 9: Relative area probability densities at $100,200, \ldots, 1500$ iterations. $G(n, 100 \Delta t)$ is dotted, $G(n, 200 \Delta t)$ is dashed, and the rest are plotted as solid lines.

to the mean curvature of the surface. We discretize our computational domain the unit cube with $\Delta x=\Delta y=\Delta z=\frac{1}{256}$, and again apply periodic boundary conditions. We maintain 64 sets of disjoint grains to track the grains through iterations. In 3D, each grain can have many more neighbors than in $2 \mathrm{D}$; thus, in general a larger number of sets $\Xi_{k}$ are necessary to keep grains within the same set well-separated.

Here, our initial condition contains 16767 total three-dimensional grains. See Figure 12 for a view of grains contained in five of the 64 sets. We take 500 iterations, at which time 853 grains remain. See Figure 11 for the evolution of the number of grains and average grain volume. We note that the dependence of $\langle v\rangle$ on $t$ is non-linear, as opposed to the relationship of $\langle a\rangle$ on $t$ in two-dimensions. In [1], the authors report that the growth kinetics exhibit power-law behavior following an initial transition phase, specifically, they report

$$
\langle v\rangle^{\frac{1}{3 n}}=c t+d
$$

where $n$ is identified as the kinetic exponent for grain growth, i.e.

$$
\langle r\rangle \approx C t^{n}
$$

gives the long term behavior of average grain size. The experimental results reported in [1] vary, reporting $1 / 4 \leq n \leq 1 / 2$ where they find $n=0.48 \pm 0.04$ using Monte Carlo methods. Our simulation data is presented in Table 3. Our results for $50 \leq t_{\min } \leq 250$ give $0.48 \leq n \leq 0.55$ which is slightly larger than what was found in [1].

Figures 12 and 13 show the same sets at later times in the evolution. Note that some grains may have swapped in or out of these sets during the evolution and thus may not be included in the visualization consistently, though large grains are swapped only rarely. Figure 14 shows a single grain from two viewpoints after 500 iterations. At this time 853 grains remain in the simulation. This grain is approximately forty grid points across in each dimension. The average effective grain radius as measured from grain volume at this time is approximately seventeen grid cells. Thus the average grain is somewhat smaller than the featured grain, but not by a significant amount. The grain appears to be very well resolved on this grid. The individual facets are apparent and are separated by sharp edges. 


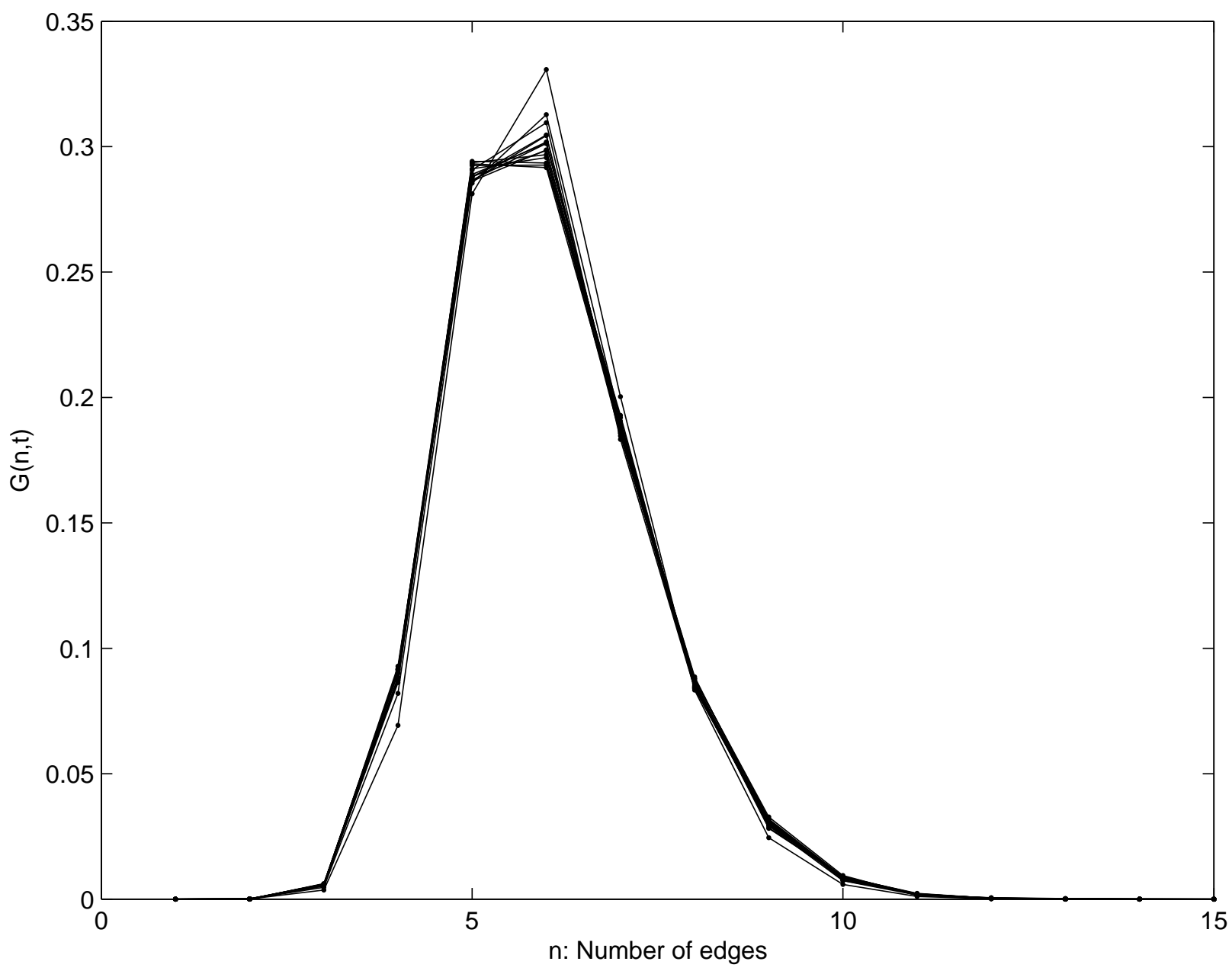

(a)

(b)

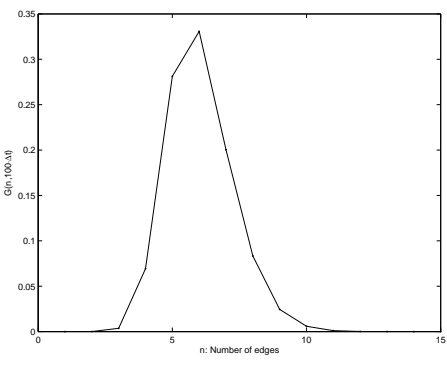

(c)

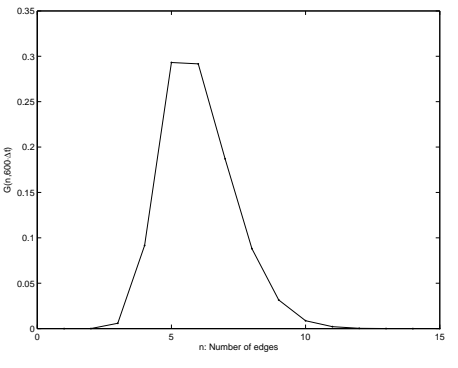

(d)

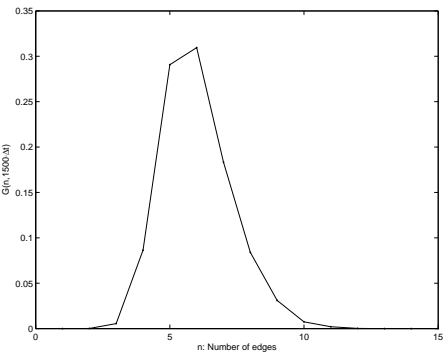

Figure 10: (a) Number of edges distribution at 100, 200, ., 1500 iterations. (b)-(d) Number of edges distribution at 100, 600, and 1500 iterations. The proportion of grains with 6 edges falls off to a minimum at 600 iterations, then rises again through the end of the simulation.

\begin{tabular}{ccc}
\hline$t_{\min }$ & $c$ & $n$ \\
\hline 10 & $2.6 \times 10^{4}$ & $.593 \pm .007$ \\
50 & $3.4 \times 10^{4}$ & $.548 \pm .005$ \\
100 & $4.4 \times 10^{4}$ & $.512 \pm .005$ \\
150 & $5.3 \times 10^{4}$ & $.487 \pm .005$ \\
200 & $5.3 \times 10^{4}$ & $.486 \pm .009$ \\
250 & $3.5 \times 10^{4}$ & $.544 \pm .018$ \\
\hline
\end{tabular}

Table 3: Fit of data from iterations in range $t_{\min } \leq t \leq 500$ to $\langle v\rangle^{1 / 3 n}=c t+d$, with $95 \%$ confidence. 

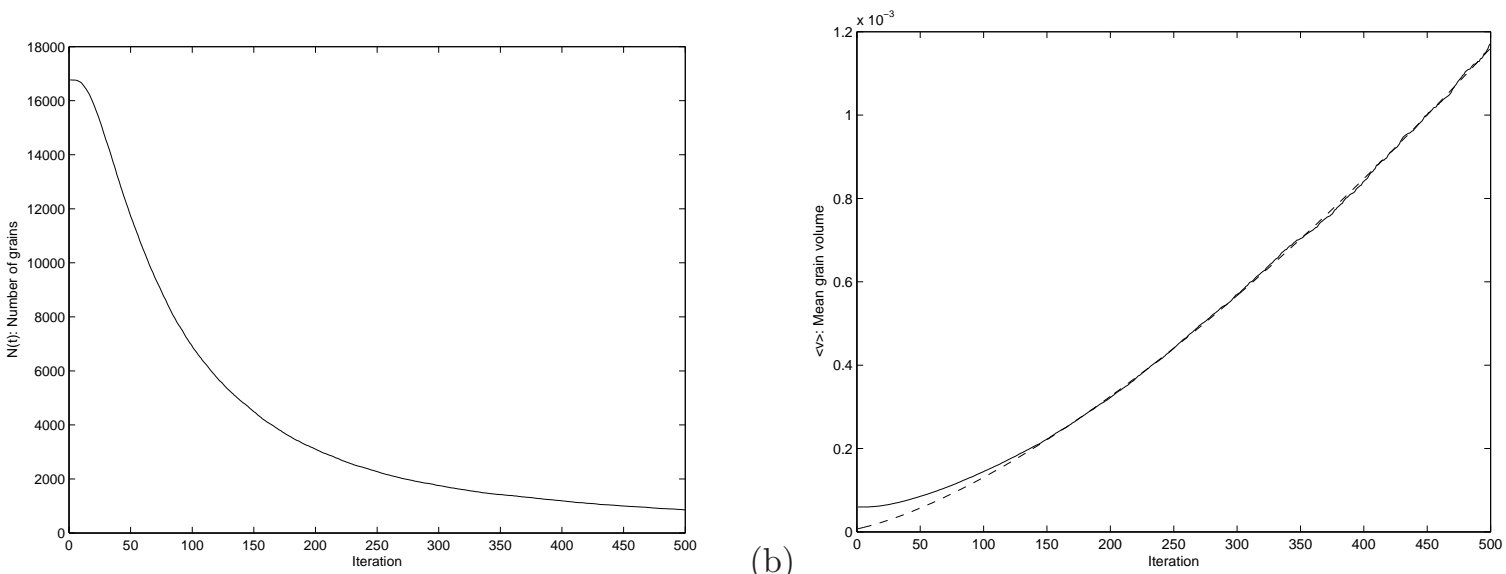

Figure 11: Three-dimensional simulation results: (a) $N(t)$, the number of grains, and (b) $\langle v\rangle$, the average grain volume (solid) plotted against $\langle v\rangle^{1 / 3 n}=c t+d$, with $t_{\min }=150$ (dashed).

Much of the statistical analysis remains to be done in three dimensions. Statistics on the fully threedimensional runs are of great interest in materials science as materials are inherently three-dimensional. Furthermore, it is also important to reconcile results from two-dimensional simulations to results from cross-sections of three-dimensional computations.

To this end, we immediately see that the character of cross-sections of three-dimensional computations is markedly different from that of the two-dimensional computations. Figure 15 shows one such cross-section. Obviously the symmetric $\left(120^{\circ}\right)$ angle condition is not expected to be preserved in crosssections, and does not appear to be in the figure. More tellingly, Figure 16 shows the relative area distribution and number of edges distribution for an ensemble of these cross-sections (containing 66437 two-dimensional grains). In agreement with data from other three dimensional simulations (e.g. [25], [1]), small grains are present in cross-section in much higher frequencies than two-dimensional simulations predict. As mentioned in [1], the greater frequency of small grains in cross-section can be partially attributed to the fact that cross-sectional planes may cut across the ends of grains that are large in three dimensions. Furthermore, the number of edges distribution (shown in Figure 16) is much flatter and wider than predicted by two-dimensional simulations.

\section{Simulations in the Presence of Bulk Energy Terms}

A simple extension to the model considered in previous sections is the inclusion of bulk energy terms:

$$
E=\sum_{k<\ell}\left(\text { length of } \Gamma_{k \ell}\right)+\sum_{k}(\text { area of phase } k) e_{k} .
$$

where $e_{k}$ denotes the bulk energy density for phase $k$. This gives rise to the following normal velocity

$$
v_{n}\left(\Gamma_{k \ell}\right)=\kappa_{k \ell}+e_{\ell}-e_{k},
$$

see, for example, $[4,17,28]$. Note that adjacent phases with equal bulk energy density terms will evolve solely by curvature, as the bulk energy contributions from each phase will cancel.

In [3], the authors present a simple modification to the two-phase algorithm to generate motion with normal speeds of the form

$$
v_{n}(\gamma)=\kappa+e
$$

In analogy, our multiphase algorithm changes in only one step. We add an additional term to the convolution step:

$$
A_{k}(x):=K_{\Delta t} * d_{k}-2(\Delta t) e_{k} .
$$

This additional set of parameters allows for the simulation of a wider class of motions. For example, an energy and normal speed of this form can be used to simulate the growth of grains on a background medium, by giving the background medium a bulk energy greater than that of the grains. We present two examples of this growth in two dimensions. 


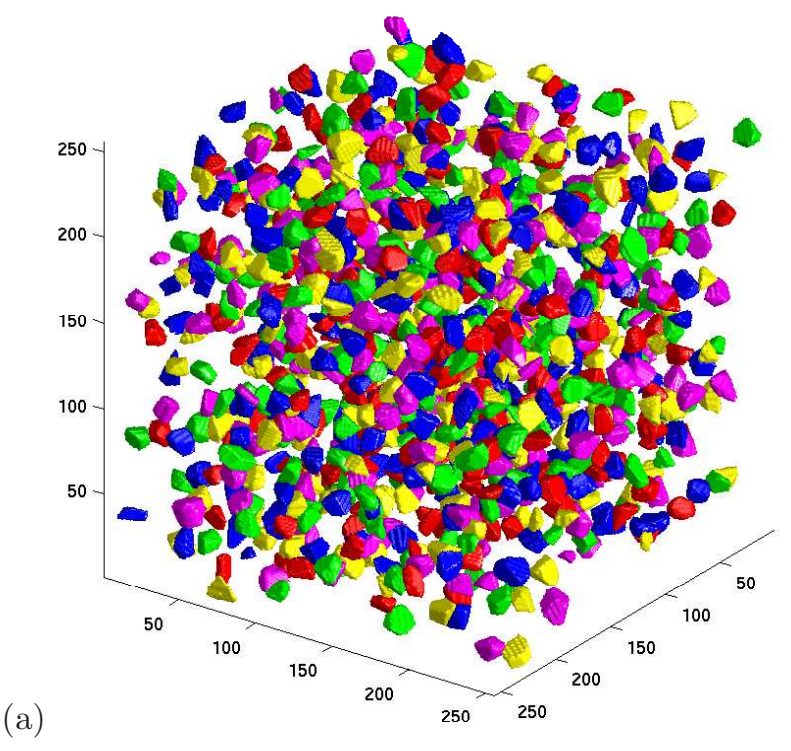

(b)
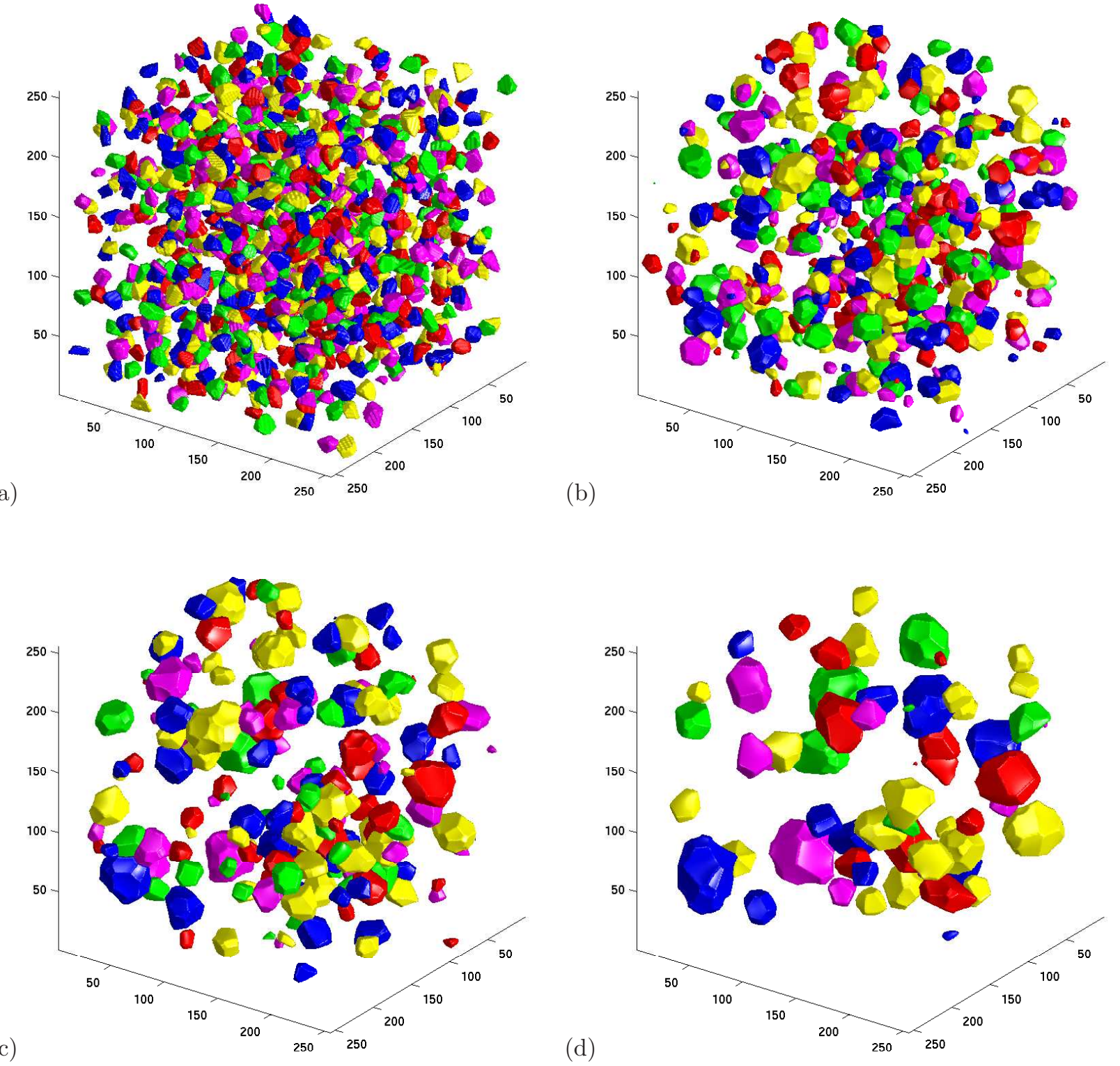

Figure 12: Each subfigure shows the grains from five sets (of sixty-four total sets) at various times in the evolution. (a) The initial condition contains 16767 total grains. (b) After 100 iterations, there are 6911 grains. (c) After 200 iterations, there are 3101 grains. (d) After 400 iterations, there are 1189 grains. 


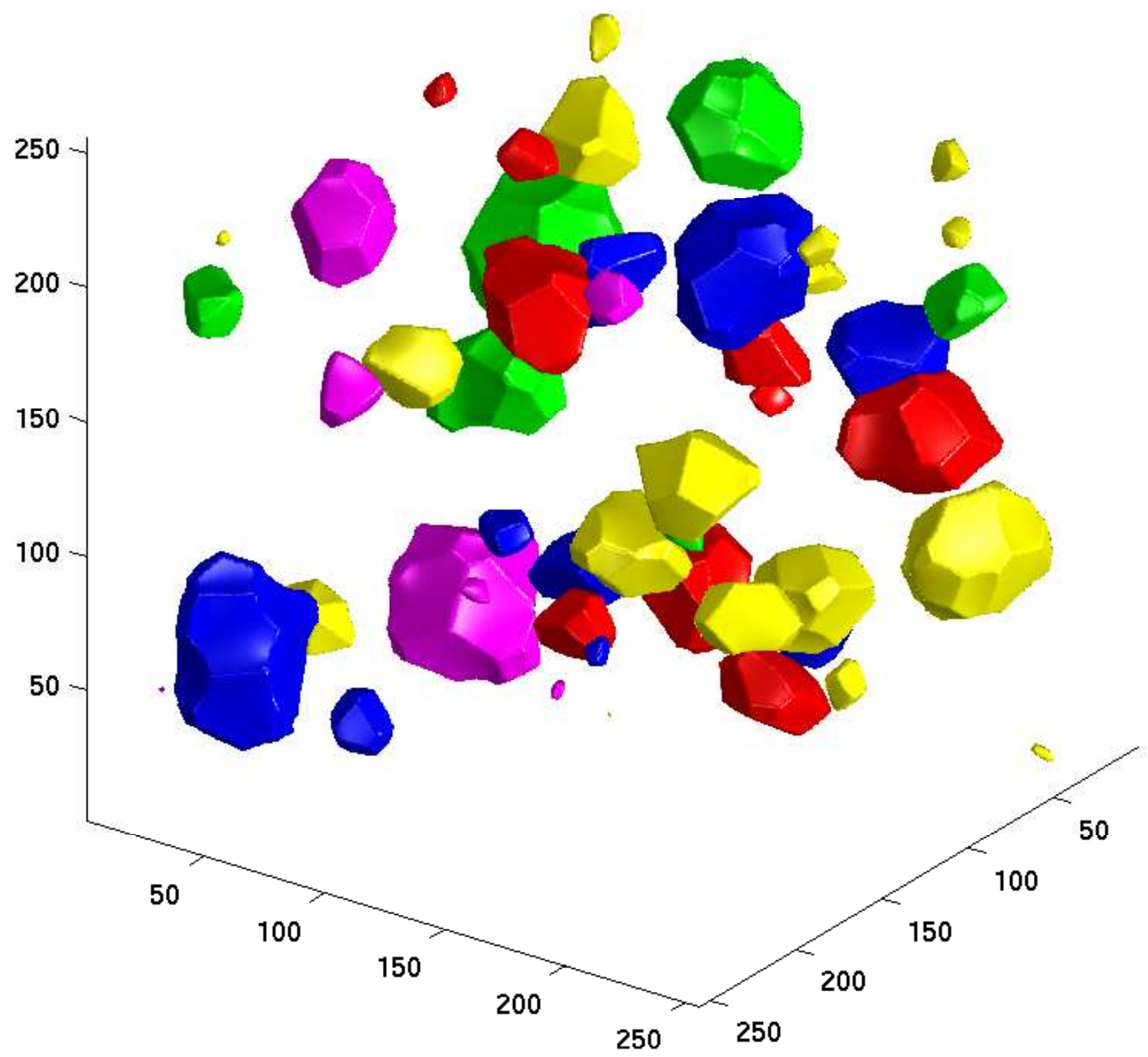

Figure 13: Grains from five level sets after 500 iterations. 853 of the initial 16767 grains remain. 

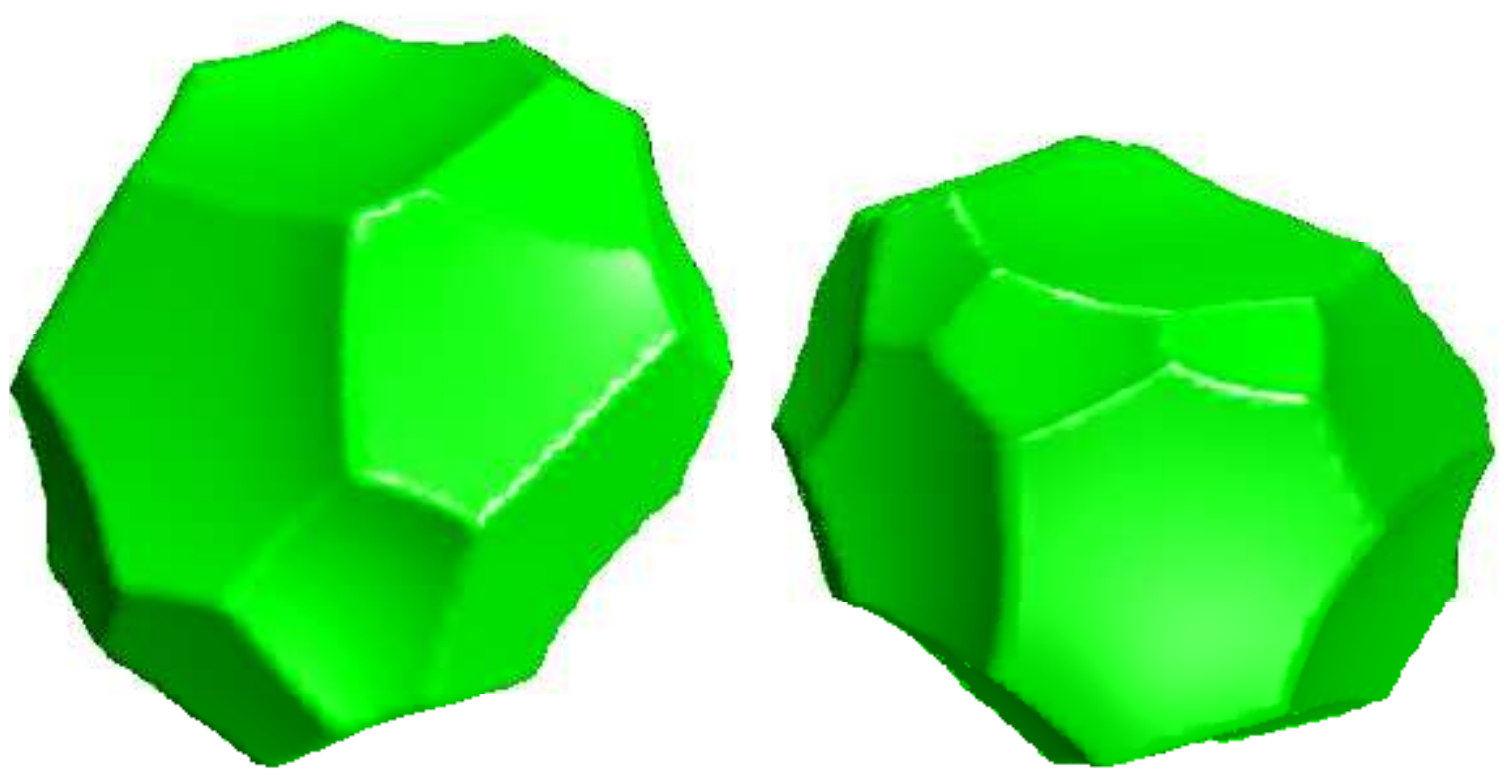

Figure 14: Two views of a single grain (corresponding to a $180^{\circ}$ rotation in the $x y$-plane) chosen from the evolution after 500 iterations. This grain is approximately forty grid cells across in each direction, slightly larger than average. The grain is very well resolved, with facets, edges, and corners all easily distinguished.

\subsection{Example 1}

We place many seeds, all with no bulk energy, near one boundary of the unit square and allow them to grow into a background medium with positive bulk energy. A simulation of this growth in two dimensions is shown in Figure 17. Where two phases meet, the interface moves solely by curvature. When a single grain meets the background phase, the normal velocity also receives a contribution from the bulk energy term. Note that in the presence of bulk energy terms, even for the $2 \mathrm{D}$ evolution, pinch-off of grains becomes possible and in fact rather frequently observed - this is a type of topological transition typically not considered (as it's believed to be absent) in front-tracking based 2D grain boundary computations in the absence of bulk energy terms.

\subsection{Example 2}

In the second example, we take the initial condition to be 100 circular grains surrounded by another material (e.g. a liquid). We take the bulk energy of the grains to be 0 and the "liquid" to have bulk energy 100/3. The coefficient of surface tension is taken to be unity (as with all of our simulations). If one considers an isolated grain of radius $r$, then it follows from Eq. (4) that

$$
\dot{r}=\frac{-1}{r}+\frac{100}{3}
$$

Therefore grains with an initial radius greater than $r_{c r i t}=0.03$ will grow, and those with initial radius less than $r_{\text {crit }}$ will shrink. One could consider this to be a highly simplified of model of solidification.

We now consider the problem in which the initial condition consists of 100 circular grains whose radii are chosen from a normal distribution with mean .03 and standard deviation .002. Our the domain is $[0,1]^{2}$ domain and we take $\Delta x=\Delta y=1 / 500$. Figure 18 shows the initial condition and the results at varying stages through 2000 iterations. Note that many circles with small initial radii have entirely disappeared. The accuracy of the numerical results for selected circles are summarized in Table 4. The predictions are generated by numerically solving Eq. (5) up to time $t=500 \Delta t$. The first and last table entries follow the largest and smallest circles in the computation, while the other entries select circles with initial radii near $r_{\text {crit }}$. The simulation data shows that the experimental value of $r_{c r i t}$ satisfies $0.3010<r_{\text {crit }}<0.3014$. 


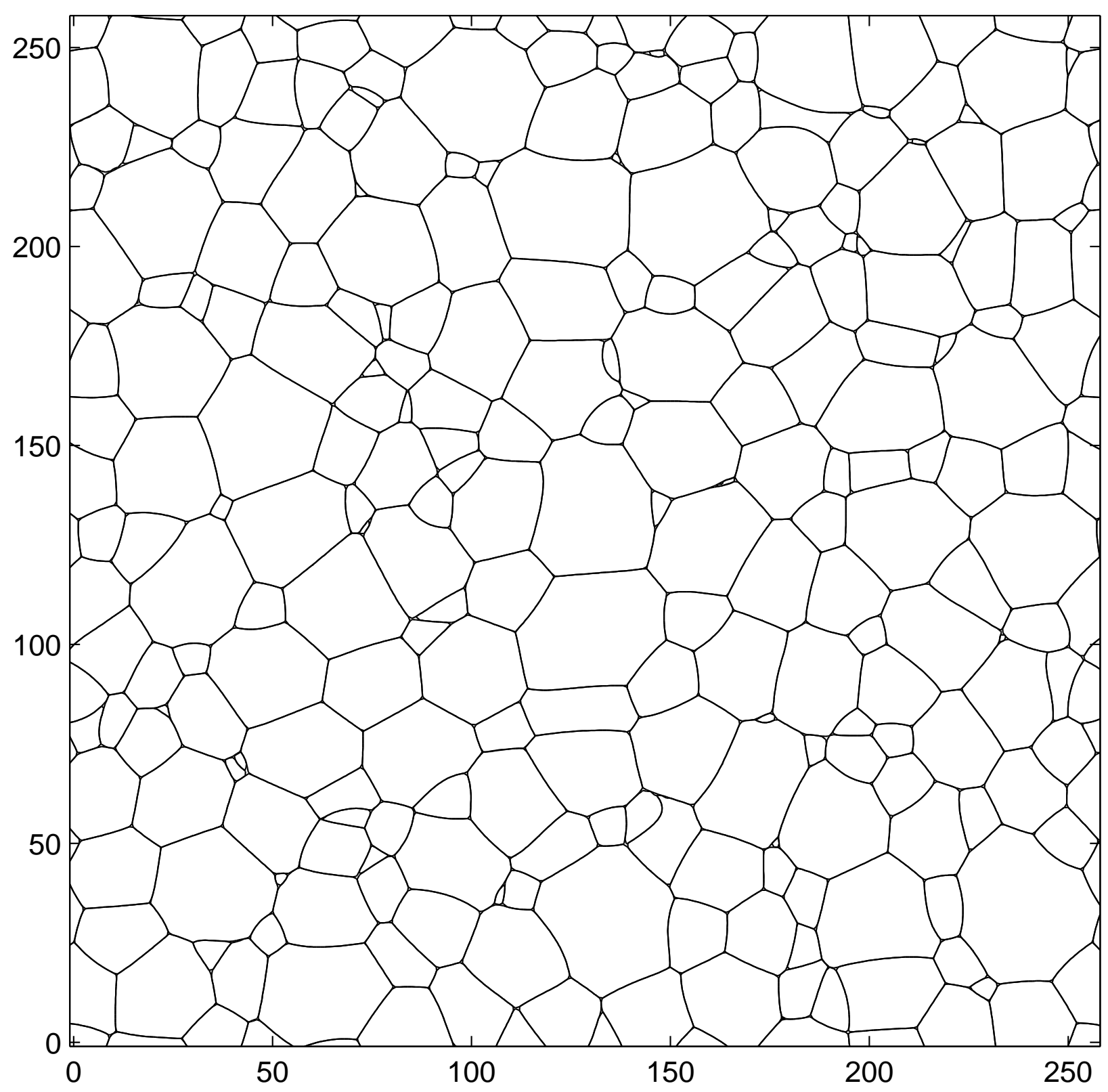

Figure 15: A cross-section of the three-dimensional evolution. In cross-section, the angles between grains do not need to be $120^{\circ}$. Many more small grains appear in cross-section than in the two-dimensional computation. Many of these small grains are small cross-sections from extremities of large three-dimensional grains, as noted in [1].

\begin{tabular}{cccc}
\hline $\begin{array}{c}\text { Initial } \\
\text { Radius }\end{array}$ & $\begin{array}{c}\text { Final } \\
\text { Radius }\end{array}$ & $\begin{array}{c}\text { Predicted } \\
\text { Radius }\end{array}$ & $\begin{array}{c}\text { Relative } \\
\text { Error }\end{array}$ \\
\hline 0.03331 & 0.04349 & 0.04379 & $0.68 \%$ \\
0.03026 & 0.03092 & 0.03145 & $1.68 \%$ \\
0.03014 & 0.03028 & 0.03080 & $1.68 \%$ \\
0.03010 & 0.02999 & 0.03059 & $1.95 \%$ \\
0.03004 & 0.02940 & 0.03024 & $2.89 \%$ \\
0.02992 & 0.02866 & 0.02952 & $2.90 \%$ \\
0.02475 & 0 & 0 & - \\
\hline
\end{tabular}

Table 4: Circular grain growth with bulk energies: Simulation and prediction. 
(a)

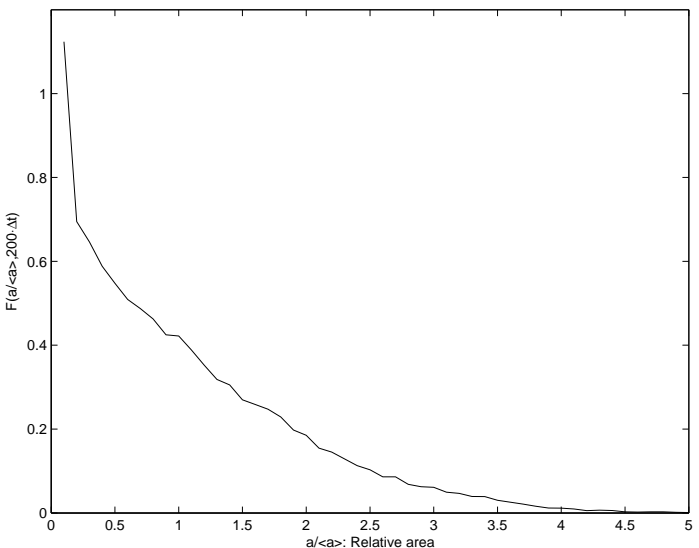

Figure 16: (a) Relative area distribution and (b) number of edges distribution from two-dimensional cross-sections of threedimensional evolution. The distribution reflects the increased number of small grains in cross-section of three-dimensional simulations as compared to two-dimensional simulations.

(a)
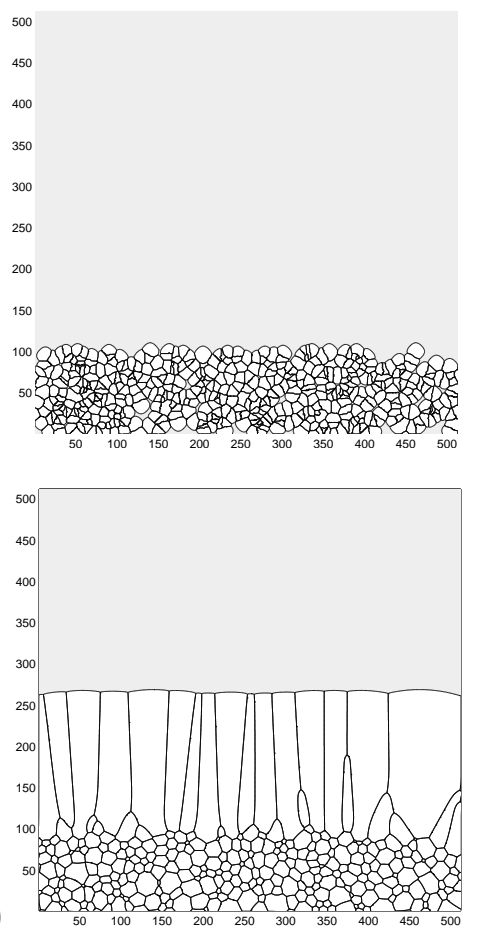

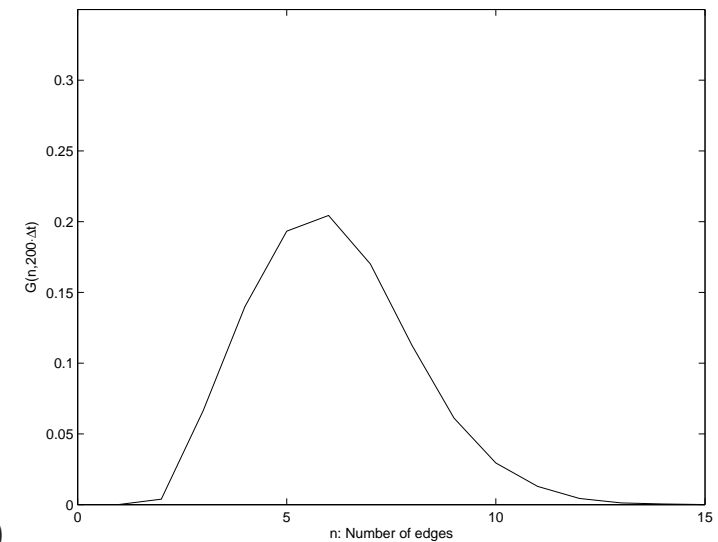

(b) .

(n)




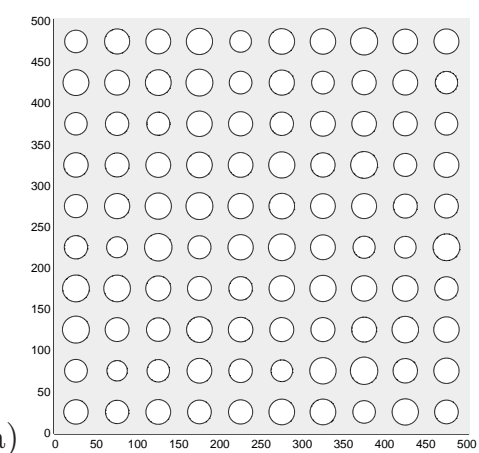

(a)

(d)

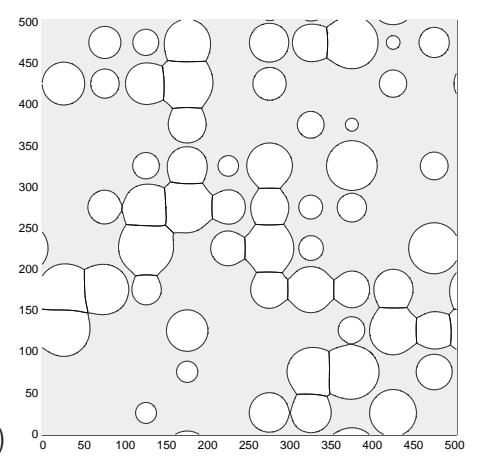

(b)

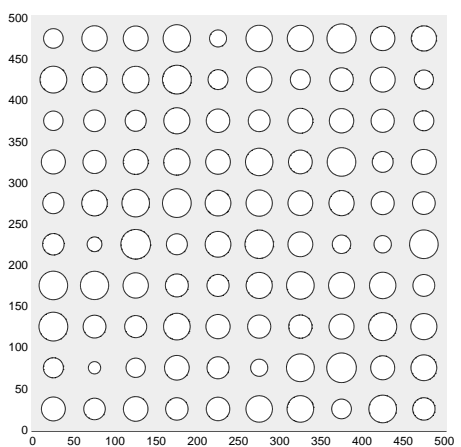

(e)

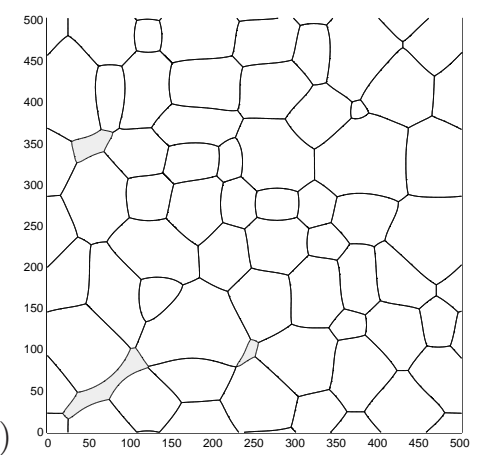

(c)

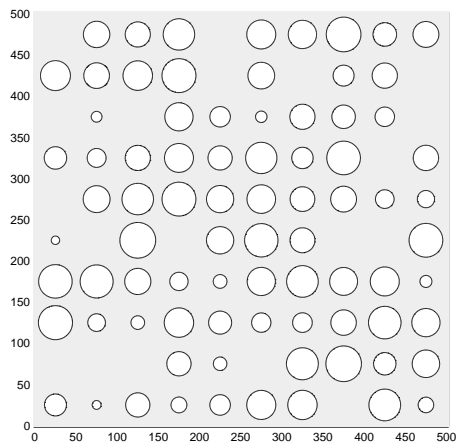

(f)

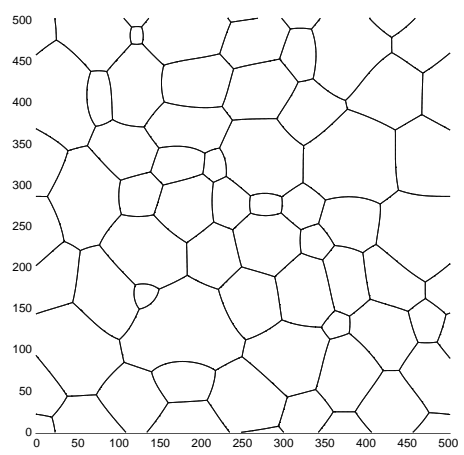

Figure 18: Circular grain growth with bulk energies at (a) initial condition, (b) 200 iterations, (c) 500 iterations, (d) 1000 iterations, (e) 1500 iterations, (f) 2000 iterations.

\section{Conclusion}

The algorithm proposed in [3] is extended here to the case of many (e.g. hundreds of thousands) phases, with allowances for bulk energies, allowing for accurate two- and three-dimensional simulations of systems larger than those obtained using other algorithms. Such large scale simulations may allow for a more complete statistical description of grain growth, particularly in three dimensions. The implicit representation of interfaces (edges in two dimensions, surfaces in three dimensions) within this model allows topological changes to occur naturally, without any explicit decision making about the nature of the change in topology or any implicit restrictions on the type of topological changes that may arise.

The model has been shown to accurately simulate well-understood motions and to predict statistics in two- and three-dimensional simulations of grain growth that are in keeping with both experiment and other simulations.

In future work, we intend to extend the model to allow for varying surface tensions, so that the normal velocity of an interface is given by

$$
v_{n}\left(\Gamma_{i j}\right)=f_{i j} \kappa_{i j}+e_{j}-e_{i}
$$

allowing for the simulation of a very general class of motions that are of interest in applications. Further refinement of the numerical techniques used should allow for even larger simulations of three-dimensional grain growth, a phenomenon which is not as well studied as the two-dimensional case. The statistics arising in this case are of great interest. They should be carefully compared to statistics arising from other simulation techniques and from available experimental results.

\section{Acknowledgments}

The authors thank David Kinderlehrer for helpful and encouraging conversations. Matt Elsey was supported by NSF DMS-0748333. Selim Esedoḡlu was supported by NSF DMS-0748333 and an Alfred P. Sloan Foundation fellowship. Peter Smereka was supported by NSF DMS-0810113. 


\section{References}

[1] Anderson, M. P., Grest, G. S., And Srolovitz, D. J. Computer simulation of normal grain growth in three dimensions. Philos. Mag. B 59, 3 (1989), 293-329.

[2] Bronsard, L., And Reitich, F. On three-phase boundary motion and the singular limit of vectorvalued Ginzburg-Landau equation. Archive for Rational Mechanics and Analysis 124 (1993), 355379.

[3] Esedō̄lu, S., Ruuth, S., and Tsai, R. Diffusion generated motion using the distance function. Tech. rep., UCLA, 2009. CAM Report 09-29.

[4] Esedō̄lu, S., And Smereka, P. A variational formulation for a level set representation of multiphase flow and area preserving curvature flow. Commun. Math. Sci. 6, 1 (2008), 125-148.

[5] FAn, D., AND L.-Q., C. Computer simulation of grain growth using a continuum field model. Acta mater. 45, 2 (1997), 611-622.

[6] Feltham, P. Grain growth in metals. Acta Metall. 5 (1957), 97-105.

[7] Garcke, H., Nestler, B., And Stoth, B. A multiphase field concept: Numerical simulations of moving phase boundaries and multiple junctions. SIAM J. Appl. Math. 60, 1 (1999), 295-315.

[8] Hillert, M. On the theory of normal and abnormal grain growth. Acta Metall. 13 (1965), 227-238.

[9] Kim, S. G., Kim, D. I., Kim, W. T., And Park, Y. B. Computer simulations of two-dimension and three-dimensional ideal grain growth. Phys. Rev. E 74 (2006), 061605.

[10] Kinderlehrer, D., Lee, J., Livshits, I., Rollett, A., And TA’Asan, S. Mesoscale simulation of grain growth. Mater. Sci. Forum 67-70 (2004), 1057-1062.

[11] Kinderlehrer, D., Livshits, I., And TA'asan, S. A variational approach to modeling and simulation of grain growth. SIAM J. Sci. Comput. 28, 5 (2006), 1694-1715.

[12] Krill III, C. E., And Chen, L.-Q. Computer simulation of 3-d grain growth using a phase-field model. Acta Mater. 50 (2002), 3057-3073.

[13] Louat, N. P. On the theory of normal grain growth. Acta Metall. 22 (1974), 721-724.

[14] Mascarenhas, P. Diffusion generated motion by mean curvature. Tech. rep., UCLA, 1992. CAM Report $92-33$.

[15] Merriman, B., Bence, J. K., And Osher, S. Motion of multiple junctions: a level set approach. J. Comput. Phys. 112, 2 (1994), 334-363.

[16] Muldins, W. Two-dimensional motion of idealized grain boundaries. J. Appl. Phys. 27, 6 (1956), 900-904.

[17] Reitich, F., And Soner, H. M. Three-phase boundary motion under constant velocities. I: The vanishing surface tension limit. Proc. R. Soc. Edin. 126A (1996), 837-865.

[18] Russo, G., And Smereka, P. A remark on computing distance functions. J. Comput. Phys. 163 (2000), 51-67.

[19] Ruuth, S. J. A diffusion-generated approach to multiphase motion. J. Comput. Phys. 145 (1998), 166-192.

[20] Ruuth, S. J. Efficient algorithms for diffusion-generated motion by mean curvature. J. Comput. Phys. 144 (1998), 603-625.

[21] Ruuth, S. J., And Merriman, B. Covolution-thresholding methods for interface motion. J. Comput. Phys. 169 (2001), 678-707.

[22] Smereka, P. The numerical approximation of a delta function with application to level set methods. J. Comput. Phys 211, 1 (2006), 77-90.

[23] Suwa, Y., Saito, Y., And Onodera, H. Parallel computer simulation of three-dimensional grain growth using the multi-phase-field model. Mater. Trans. 49, 4 (2008), 704-709.

[24] Tsai, Y.-H. R., Cheng, L.-T., Osher, S., and ZhaO, H.-K. Fast sweeping algorithms for a class of hamilton-jacobi equations. SIAM J. Numer. Anal. 41, 2 (2003), 673-694.

[25] Wakai, F., Enomoto, N., and Ogawa, H. Three-dimensional microstructural evolution in ideal grain growth-general statistics. Acta Mater. 48 (2000), 1297-1311. 
[26] Zhang, X., Chen, J.-S., And Osher, S. A multiple level set method for modeling grain boundary evolution of polycrystalline materials. Tech. rep., UCLA, 2006. CAM Report 06-69.

[27] ZhaO, H.-K. A fast sweeping method for eikonal equations. Math. Comput. 74, 250 (2004), 603-627.

[28] Zhao, H.-K., Chan, T., Merriman, B., and Osher, S. A variational level set approach to multiphase motion. J. Comput. Phys. 127 (1996), 179-195. 